ARTICLE

https://doi.org/10.1038/s41467-019-12610-x

\title{
ZC3H18 specifically binds and activates the BRCA1 promoter to facilitate homologous recombination in ovarian cancer
}

Arun Kanakkanthara (1) 1,2, Catherine J. Huntoon ${ }^{1}$, Xiaonan Hou $^{3}$, Minzhi Zhang ${ }^{3}$, Ethan P. Heinzen ${ }^{4}$, Daniel R. O'Brien ${ }^{4}$, Ann L. Oberg (1) ${ }^{4}$, S. John Weroha ${ }^{3}$, Scott H. Kaufmann (1) ${ }^{1,2}$ \& Larry M. Karnitz ${ }^{1,2 \star}$

Reduced BRCA1 expression causes homologous recombination (HR) repair defects in highgrade serous ovarian cancers (HGSOCs). Here, we demonstrate that BRCA1 is transcriptionally activated by a previously unknown function of $\mathrm{ZC} 3 \mathrm{H} 18$. We show that $\mathrm{ZC} 3 \mathrm{H} 18$ is a DNA-binding protein that interacts with an E2F site in the BRCA1 promoter where it facilitates recruitment of E2F4 to an adjacent E2F site to promote BRCA1 transcription. Consistent with $\mathrm{ZC} 3 \mathrm{H} 18$ role in activating BRCA1 expression, $\mathrm{ZC} 3 \mathrm{H} 18$ depletion induces BRCA1 promoter methylation, reduces BRCA1 expression, disrupts $\mathrm{HR}$, and sensitizes cells to DNA crosslinkers and poly(ADP-ribose) polymerase inhibitors. Moreover, in patient-derived xenografts and primary HGSOC tumors, ZC3H18 and E2F4 mRNA levels are positively correlated with BRCA1 mRNA levels, further supporting ZC3H18 role in regulating BRCA1. Given that $Z C 3 H 18$ lies within 16q24.2, a region with frequent copy number loss in HGSOC, these findings suggest that ZC3H18 copy number losses could contribute to HR defects in HGSOC.

\footnotetext{
${ }^{1}$ Division of Oncology Research, Mayo Clinic, Rochester, MN, USA. ${ }^{2}$ Department of Pharmacology, Mayo Clinic, Rochester, MN, USA. ${ }^{3}$ Division of Medical Oncology, Mayo Clinic, Rochester, MN, USA. ${ }^{4}$ Division of Biomedical Statistics and Informatics, Mayo Clinic, Rochester, MN, USA.

*email: karnitz.larry@mayo.edu
} 
$\mathrm{H}$ omologous recombination (HR) is a high-fidelity DNA repair mechanism that requires the sequential activities of a series of proteins, including BRCA1 and BRCA2 tumor suppressors ${ }^{1}$. Defects in HR are a defining feature of high-grade serous ovarian cancers (HGSOCs), the most common and lethal ovarian cancer subtype ${ }^{1,2}$. The most frequent causes of $\mathrm{HR}$ defects are deleterious mutations in BRCA1 and BRCA2 (ref. ${ }^{1}$ ), which are associated with increased response rates to platinumbased therapies, enhanced disease-free survival, and improved overall survival $^{1-3}$. HGSOCs with deleterious $B R C A 1 / 2$ mutations are also sensitive to poly(ADP-ribose) polymerase (PARP) inhibitors ${ }^{1,2}$.

Notably, many HGSOCs have HR defects despite a lack of mutations in BRCA1/2 and other known DNA repair genes ${ }^{4}$. A substantial fraction of those are due to reduced BRCA1 transcription, which is associated with HR defects in HGSOCs ${ }^{5-8}$. Two known mechansisms that cause reduced BRCA1 expression include (1) hypermethylation of the BRCA1 promoter, which occurs in $8-15 \%$ of HGSOCs; $9,10,11$ and (2) mutational inactivation of CDK12 (ref. ${ }^{11}$ ), an RNA polymerase II C-terminal domain (CTD) kinase that regulates the transcription of $B R C A 1$ and other genes ${ }^{12,13}$. Additionally, BRCA1 transcription is controlled by a complex array of transcription factors, coactivators, and corepressors that interact with the BRCA1 promoter ${ }^{14-16}$. However, a complete understanding of the transcriptional regulation of BRCA1 is lacking.

Here, we report on a previously uncharacterized mode of BRCA1 transcriptional regulation. We show that BRCA1 transcription is regulated by $\mathrm{ZC} 3 \mathrm{H} 18$, which we demonstrate has a previously unknown biochemical function: $\mathrm{ZC} 3 \mathrm{H} 18$ is a DNA-binding protein that interacts with an E2F site in the $B R C A 1$ promoter and that activates transcripton. Accordingly, these studies expand the known roles for $\mathrm{ZC} 3 \mathrm{H} 18$, which was previously shown to participate in RNA processing by mediating mRNA export, degradation, and transcription of a subset of protein-coding genes through its association with the mRNA cap-binding complex and the nuclear exosome-targeting complex ${ }^{17-20}$. This study also shows that $\mathrm{ZC} 3 \mathrm{H} 18$ binding to an E2F site in the BRCA1 promoter enhances the association of E2F4 with an adjacent E2F site to activate BRCA1 transcription. Consistent with these observations, ZC3H18 and E2F4 mRNA levels correlated with BRCA1 mRNA levels in primary human HGSOC tumors and patient-derived xenograft (PDX) models.

Collectively, these results discover an additional biochemical function for ZC3H18; uncover a uncharacterized mechanism of $B R C A 1$ transcriptional regulation; and because $\mathrm{ZC} 3 \mathrm{H} 18$ is located in a region (chromosome 16q24.2) of recurrent copy number loss in $\mathrm{HGSOC}^{21,22}$, suggest that reduced $\mathrm{ZC} 3 \mathrm{H} 18$ levels may be an unrecognized contributor to diminished BRCA1 expression and HR defects in HGSOC.

\section{Results}

ZC3H18 depletion induces an HR defect and DNA damage sensitivity. Copy number losses in chromosomal region 16q24.2 are a common event in HGSOC (Supplementary Fig. 1a). Indeed, some studies have reported 16q24.2 loss to be among the most frequent copy number variation in $\mathrm{HGSOC}^{21,22}$, raising the possibility that genes located within this region could impact HR. To assess the potential role of genes in this region in $\mathrm{HR}$, we conducted an siRNA screen of known protein-coding genes at 16q24.2 using OVCAR-8 cells that have a genomically integrated DR-GFP ${ }^{23}$ reporter construct ${ }^{12}$. Among the 16 protein-coding genes at 16q24.2, depletion of $\mathrm{ZC} 3 \mathrm{H} 18$ had the largest effect on HR (Supplementary Fig. 1b).
In further experiments, we confirmed that $\mathrm{ZC} 3 \mathrm{H} 18$ plays a role in HR by showing that two independent siRNAs reduced ZC3H18 protein, disrupted DR-GFP recombination (Fig. 1a), and blocked the formation of RAD51 foci (Fig. 1b), a key event in HR repair, without disrupting the cell cycle (Supplementary Fig. 1c). Conversely, expression of an siRNA-resistant ZC3H18 rescued the $\mathrm{HR}$ defect in $\mathrm{ZC} 3 \mathrm{H} 18$-depleted cells (Fig. 1c), indicating that the siRNA effect is due to $\mathrm{ZC} 3 \mathrm{H} 18$ depletion. We also demonstrated that $\mathrm{ZC} 3 \mathrm{H} 18$-depleted ovarian cancer cell lines (Supplementary Fig. 2a) were sensitive to the DNA crosslinkers cisplatin and melphalan as well as the PARP inhibitors olaparib and veliparib in culture (Fig. 1d, e; and Supplementary Fig. 2b). Consistent with the cell culture results, shRNA-mediated ZC3H18 depletion (Supplementary Fig. 2c) also sensitized xenografted OVCAR-8 cells to olaparib in mice treated with this PARPi (Fig. 1f). Collectively, these results demonstrate that ZC3H18, a gene located in a chromosomal region frequently deleted in HGSOC, is essential for $\mathrm{HR}$ and that $\mathrm{ZC} 3 \mathrm{H} 18$ depletion sensitizes ovarian cancer cells to platinum agents and PARP inhibitors.

ZC3H18 depletion reduces BRCA1, which drives the HR defect. Because $\mathrm{ZC} 3 \mathrm{H} 18$ was previously shown to regulate gene expression through its effects on RNA metabolism ${ }^{17,18,19,20}$, we asked whether ZC3H18 depletion affected expression of genes associated with HR by RNA-seq (Supplementary Data 1). A KEGG pathway analysis ${ }^{24}$ showed that multiple HR-associated genes were downregulated (Supplementary Fig. 3), with BRCA1 among the most highly reduced by ZC3H18 depletion (Supplementary Data 1). Because defects in BRCA1 are the most frequent cause of HR deficiency and BRCA1 is a key regulator of $\mathrm{HR}^{1}$, we focused the present studies on BRCA1. Consistent with the RNAseq analysis, ZC3H18 depletion profoundly decreased BRCA1 mRNA (Fig. 2b; Supplementary Fig. 4a) and protein levels (Fig. 2a) in multiple ovarian cancer cell lines and in xenografted OVCAR-8 cells (Supplementary Fig. 2c). Moreover, expression of siRNA-resistant ZC3H18 restored BRCA1 mRNA (Fig. 2c) and protein levels (Supplementary Fig. 4b) in ZC3H18 siRNAtransfected cells confirming that ZC3H18 facilitates accumulation of BRCA1 mRNA and protein. Finally, because multiple HRassociated genes were downregulated by $\mathrm{ZC} 3 \mathrm{H} 18$ depletion (Supplementary Data 1 and Supplementary Fig. 3), we next asked whether the loss of BRCA1 was a major contributor to the HR defect caused by ZC3H18 depletion. As shown in Fig. 2d, heterologous expression of HA-tagged BRCA1 (Supplementary Fig. 4c) substantially restored HR, thus showing that the loss of BRCA1 is a major driver of the HR defect caused by ZC3H18 depletion.

ZC3H18 depletion causes BRCA1 promoter hypermethylation. Given that ZC3H18 was previously shown to affect RNA splicing and degradation ${ }^{17-20}$, we next assessed whether $\mathrm{ZC} 3 \mathrm{H} 18$ regulates BRCA1 levels by altering these BRCA1 mRNA processing events. In ZC3H18 siRNA-transfected ovarian cancer cells, we found no evidence of alternative BRCA1 RNA splicing using a PCR-based method ${ }^{25}$ (Supplementary Fig. 5a). Additionally, BRCA1 mRNA half-life was not affected by ZC3H18 depletion (Supplementary Fig. 5b). Similarly, depletion of ZCCHC8, which is a component of the $\mathrm{ZC} 3 \mathrm{H} 18$ cap-binding complex that mediates RNA degradation ${ }^{19}$, did not alter BRCA1 mRNA levels (Supplementary Fig. 5c). These results suggest that $\mathrm{ZC} 3 \mathrm{H} 18$ mediates BRCA1 expression through a mechanism that differs from its previously identified functions.

Because BRCA1 is frequently silenced by promoter hypermethylation $6,8,26,27$, we next examined whether $\mathrm{ZC} 3 \mathrm{H} 18$ 
a

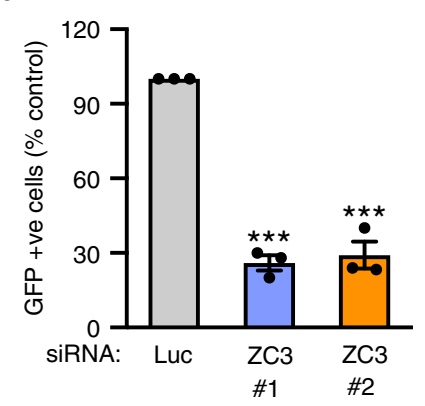

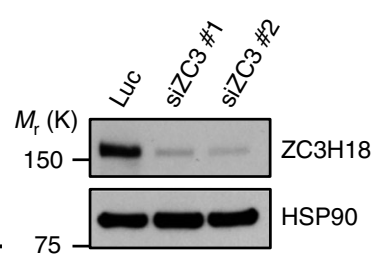

b

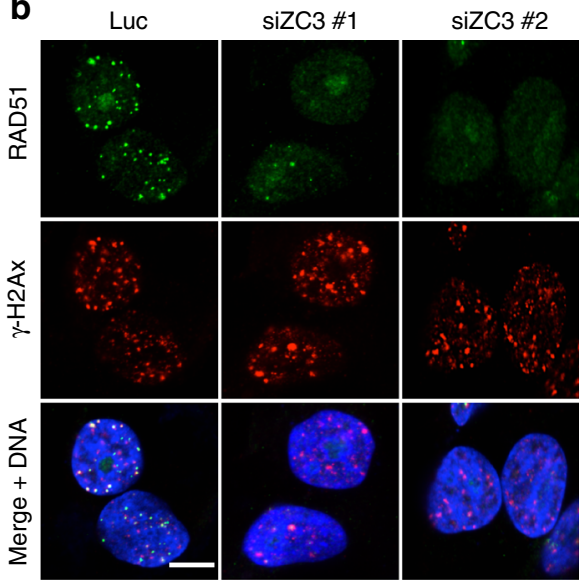

C

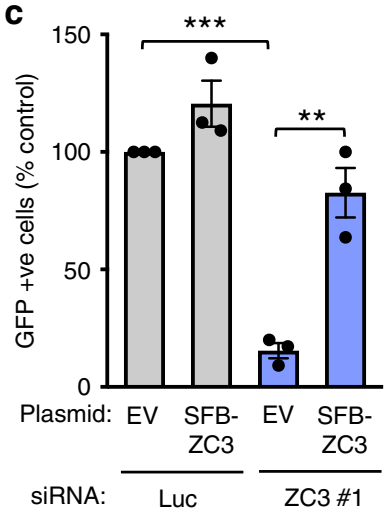

d

OVCAR-8

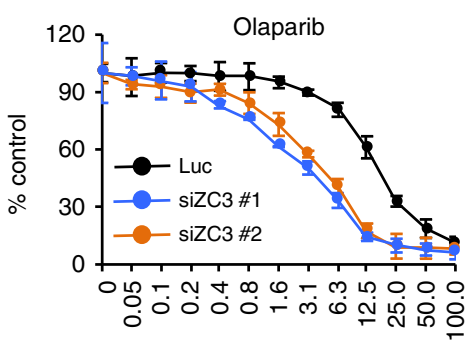

Concentration $(\mu \mathrm{M})$

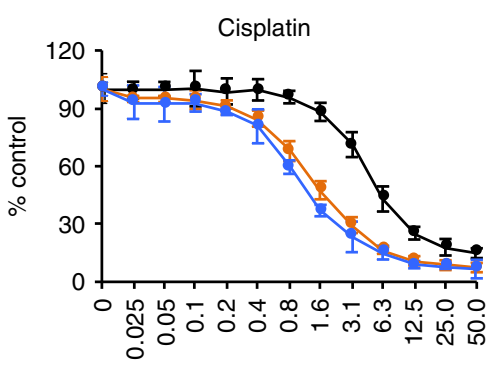

Concentration $(\mu \mathrm{M})$

siRNA: $\frac{\text { Luc }}{\text { SFB- }} \frac{\text { ZC3\#1 }}{\text { SFB- }}$

Plasmid: EV ZC3 EV ZC3

$M_{\mathrm{r}}(\mathrm{K})$

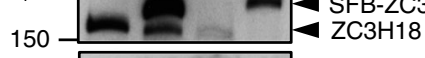

HSP9O

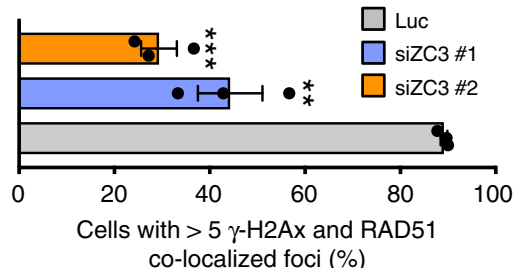

e
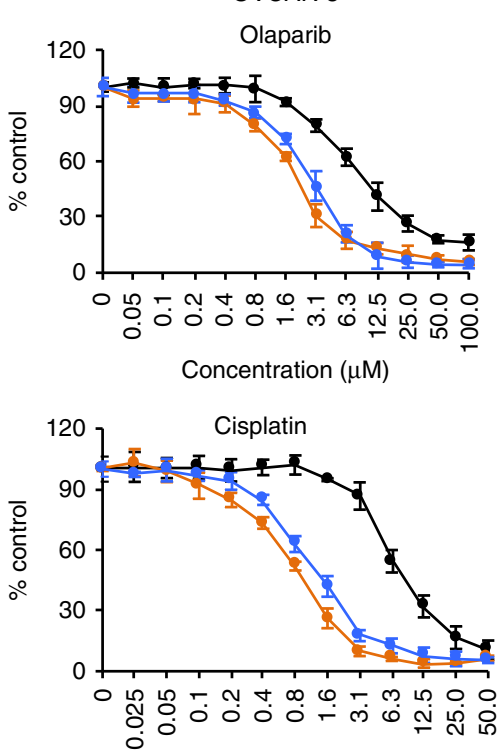

Concentration $(\mu \mathrm{M})$ f

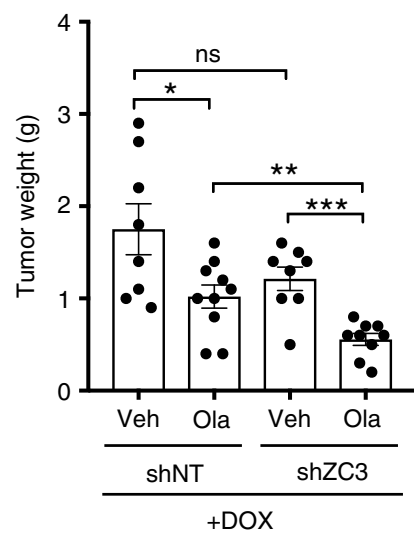

depletion affected $B R C A 1$ promoter $C p G$ methylation and the recruitment of DNA methyltransferase 1 (DNMT1), the prototypical DNA methyltransferase, to the BRCA1 promoter. As previously reported ${ }^{28}$, bisulfite sequencing revealed that $10-20 \%$ of the BRCA1 promoter CpG sites were methylated in control siRNA-transfected OVCAR-8 cells (Fig. 3a). In contrast, ZC3H18 depletion with two independent siRNAs increased BRCA1 promoter methylation of these sites to $\sim 50 \%$ (Fig. 3a). Consistent with these results, chromatin immunoprecipitation (ChIP) assays showed that $\mathrm{ZC} 3 \mathrm{H} 18$ depletion increased the accumulation of DNMT1 on the BRCA1 promoter in two separate HGSOC cell lines, OVCAR-8 and PEA1 (Fig. 3b), without altering DNMT1 expression (Supplementary Fig. 5d). Conversely, treatment of the
ZC3H18-depleted cells with the DNA methylation inhibitor 5-aza-2'-deoxycytidine (5-aza-dC) restored BRCA1 mRNA levels without affecting $\mathrm{ZC} 3 \mathrm{H} 18 \mathrm{mRNA}$ levels (Fig. 3c, d), demonstrating that BRCA1 promoter methylation induced by $\mathrm{ZC} 3 \mathrm{H} 18$ depletion reduces $B R C A 1$ expression.

ZC3H18 depletion promotes E2F1-mediated repression of BRCA1. To determine how $\mathrm{ZC} 3 \mathrm{H} 18$ deficiency induces $B R C A 1$ promoter methylation, we first asked if ZC3H18 associates with the BRCA1 promoter. ChIP of endogenous or overexpressed ZC3H18 demonstrated that ZC3H18 associates with the BRCA1 promoter in multiple ovarian cancer cell lines (Fig. 4a, b), raising 
Fig. $1 \mathrm{ZC} 3 \mathrm{H} 18$ depletion disrupts HR. a Analysis of HR efficiency. OVCAR-8-DR-GFP cells were transfected with pC $\beta A S c e l$ plasmid and control luciferase (Luc) or independent ZC3H18 siRNAs (siZC3 \#1 and siZC3 \#2). Cells were analyzed for GFP fluorescence (left panel) and immunoblotted for ZC3H18 and HSP9O (right panel) $72 \mathrm{~h}$ later. HR efficiencies were normalized to cells with Luc siRNA. b OVCAR-8 cells were transfected with control Luc or

ZC3H18 siRNAs and co-immunostained with RAD51 and $\gamma$-H2AX antibodies (upper panel shows representative image). Cells with $\geq 5$ colocalized RAD51 and $\gamma-\mathrm{H} 2 \mathrm{AX}$ foci were quantitated (bottom panel) $72 \mathrm{~h}$ later. c DR-GFP-OVCAR-8 cells were transfected with pC $\beta A S c e l$ plasmid and control Luc siRNA or ZC3H18 siRNA \#1 plus empty vector (EV) or siRNA-resistant ZC3H18 plasmid (SFB-ZC3H18). Cells were analyzed for GFP (left panel) and immunoblotted for ZC3H18 and HSP9O (right panel shows representative immunoblot) 48 h later. d, e OVCAR-8 (d) and OVCAR-5 (e) cells were transfected with control Luc or ZC3H18 siRNAs. $48 \mathrm{~h}$ later, cells were immunoblotted for ZC3H18 and HSP90 (Supplementary Fig. 2a) or were re-plated, treated with cisplatin for 3 days or olaparib for 7 days, and analyzed by MTS assay. $\mathbf{f}$ OVCAR-8 cells with stably transduced, doxorubicin-inducible non-targeting shRNA (shNT) or ZC3H18 shRNA (shZC3) were innoculated into mice. 5 days later, shRNAs were induced by feeding doxycline chow, and mice were treated with vehicle (Veh) or olaparib (Ola). After 4 weeks of treatment, tumor weights were determined. Shown are means \pm SEM from three independent experiments in $\mathbf{a}$, $\mathbf{b}$ (bottom panel), and $\mathbf{c}$. Representative images of three independent experiments are shown in $\mathbf{b}$ (top panel).In all experiments, $\geq 100$ cells were counted per experiment. Scale bar, $10 \mu \mathrm{m}$. Graphs in $\mathbf{d}$ and $\mathbf{e}$ represent one of three independent experiments that gave similar results. Error bars are standard error of triplicate wells from an individual representative experiment. Shown are means \pm SEM from 8 to 10 mice per group in $\mathbf{f}$. ns, not significant, ${ }^{\star} p<0.05,{ }^{\star \star} p<$ $0.01,{ }^{* \star *} p<0.001$, unpaired Student's $t$-test. Representative immunoblots in $\mathbf{a}$ and $\mathbf{c}$ are provided from three independent experiments. Unprocessed blots are in source data file

the possibility that ZC3H18 might affect the binding of transcription factors that regulate BRCA1 expression.

E2F family members are key transcription factor regulators of the BRCA1 gene that can either activate or repress transcription, depending on the family member that binds the promoter ${ }^{29-31}$. E2F1 was previously shown to activate BRCA1 transcription in breast cancer and other cell types ${ }^{30,32,33}$. Accordingly, we asked whether $\mathrm{ZC} 3 \mathrm{H} 18$ affects $B R C A 1$ expression by reducing E2F1 binding to the BRCA1 promoter, an event predicted to reduce $B R C A 1$ expression. Suprisingly, however, ZC3H18 depletion greatly increased E2F1 binding to the BRCA1 promoter (Fig. 4c, d), suggesting that E2F1 was not activating BRCA1 transcription in these cells. Consistent with this possibility, E2F1 depletion did not reduce BRCA1 expression in ovarian cancer cell lines (Fig. 4e; Supplementary Fig. 6a) or in freshly isolated HGSOC tumors from two different mouse PDX models (Supplementary Fig. 6b, c) using primers that are specific for human BRCA1 (Supplementary Fig. 6c), thus demonstrating that E2F1 does not play a major role in BRCA1 transactivation in HGSOC.

Although E2F1 is generally considered to be a transcriptional activator, it can also repress transcription in some settings by recruiting the DNA methyltransferase DNMT1 to promoters ${ }^{34}$. Given that $\mathrm{ZC} 3 \mathrm{H} 18$ depletion caused BRCA1 promoter methylation and E2F1 recruitment to the BRCA1 promoter, we reasoned that E2F1 might repress BRCA1 expression in ovarian cancer cells by recruiting DNMT1 when ZC3H18 was depleted. Consistent with this possibility, we found that $\mathrm{E} 2 \mathrm{~F} 1$ was required for DNMT1 recruitment to the BRCA1 promoter (Fig. 4f). In additional experiments, E2F1 depletion restored BRCA1 expression in ZC3H18-depleted cells (Fig. 4g), further demonstrating that E2F1 mediates BRCA1 repression in this setting.

These findings suggested that the role of E2F1 in BRCA1 transcriptional regulation might differ between ovarian cancer cells, where E2F1 represses BRCA1 transcription (Fig. 4f, g), and breast cancer cells, in which E2F1 promotes BRCA1 transcription ${ }^{30,32,33}$. To further evaluate this possibility, we examined the effect of depleting E2F1 in MDA-MB-231 breast cancer cells. Consistent with previous reports in breast cancer cells, we found that E2F1 depletion reduced BRCA1 expression in MDA-MB-231 cells (Supplementary Fig. 7a). We next assessed the possibility that ZC3H18 might affect E2F1 and DNMT1 differently in ovarian and breast cancer cells. Indeed, ZC3H18 depletion enhanced E2F1 binding to DNMT1 in ovarian cancer but not in breast cancer cells (Supplementary Fig. 7b, c), suggesting that loss of ZC3H18 leads to E2F1-DNMT1 repressor complex formation in ovarian cancer cells. Despite the different roles of E2F1 in ovarian and breast cancer cells, ZC3H18 depletion reduced BRCA1 levels in both cell lines (Supplementary Fig. 7a). Collectively, these results demonstrate that although ZC3H18 depletion reduces BRCA1 expression in both ovarian and breast cancer cells, the mechanisms underlying $\mathrm{ZC} 3 \mathrm{H} 18$ regulation of $B R C A 1$ differs in the two cells types, with $\mathrm{ZC} 3 \mathrm{H} 18$ regulating the recruitment of E2F1 and DNMT1 to the BRCA1 promoter to repress transcription in ovarian cancer cells.

ZC3H18 facilitates E2F4 binding to the BRCA1 promoter. Because multiple E2F family members have been reported to regulate $B R C A 1$ transcription in a variety of cell line models $^{15,30,32,33}$, we next explored the roles of the seven other known family members (E2F2-E2F8) in control and ZC3H18depleted OVCAR-8 cells. These studies showed that (1) E2F4 was the only E2F family member that affected BRCA1 expression and (2) co-depletion of $\mathrm{E} 2 \mathrm{~F} 4$ and $\mathrm{ZC} 3 \mathrm{H} 18$ did not further suppress BRCA1 mRNA levels (Fig. 5a), suggesting that E2F4 and ZC3H18 are in the same pathway. Additional studies showed that E2F4 depletion with two independent siRNAs reduced BRCA1 mRNA and protein levels in OVCAR-8, PEA1, PEO1, and PEO4 cells without affecting ZC3H18 levels (Fig. 5b; Supplementary Fig. 8a, b). Similarly, E2F4 depletion also reduced BRCA1 expression in short-term ex vivo cultures of HGSOC tumors freshly isolated from three different PDX models (Fig. 5c).

Because the results in Fig. 5a and Supplementary Fig. 8a suggested that $\mathrm{ZC} 3 \mathrm{H} 18$ and $\mathrm{E} 2 \mathrm{~F} 4$ regulate $B R C A 1$ through the same pathway, we next asked whether ZC3H18 and E2F4 affected one another's interaction with the BRCA1 promoter using ChIP. ZC3H18 depletion reduced E2F4 occupancy on the BRCA1 promoter but did not affect E2F4 expression (Fig. 5d; Supplementary Fig. 8c, d). In contrast, E2F4 depletion did not alter ZC3H18 binding to the BRCA1 promoter (Supplementary Fig. 8e), demonstrating that $\mathrm{ZC} 3 \mathrm{H} 18$ enhances the recruitment of E2F4 to the BRCA1 promoter but not vice versa. These findings also suggested that E2F4 might contribute to the effects of $\mathrm{ZC} 3 \mathrm{H} 18$ on BRCA1 expression. Consistent with this idea, E2F4 siRNAs increased E2F1 (Fig. 5e) and DNMT1 (Fig. 5f) recruitment to the BRCA1 promoter, blocked HR (Fig. 5g), and sensitized cells to the PARP inhibitor olaparib (Fig. 5h) without disrupting the cell cycle (Supplementary Fig. 8f). Together, these results demonstrate that $\mathrm{ZC} 3 \mathrm{H} 18$ enhances $\mathrm{E} 2 \mathrm{~F} 4$ binding to the $B R C A 1$ promoter, which concomitantly reduces binding of E2F1 and DNMT1 to the promoter and promotes BRCA1 transcription.

ZC3H18 and E2F4 bind adjacent E2F sites. Two key E2F binding sites, E2FA and $\mathrm{E} 2 \mathrm{FB}^{15,33}$, have been identified in the 
a
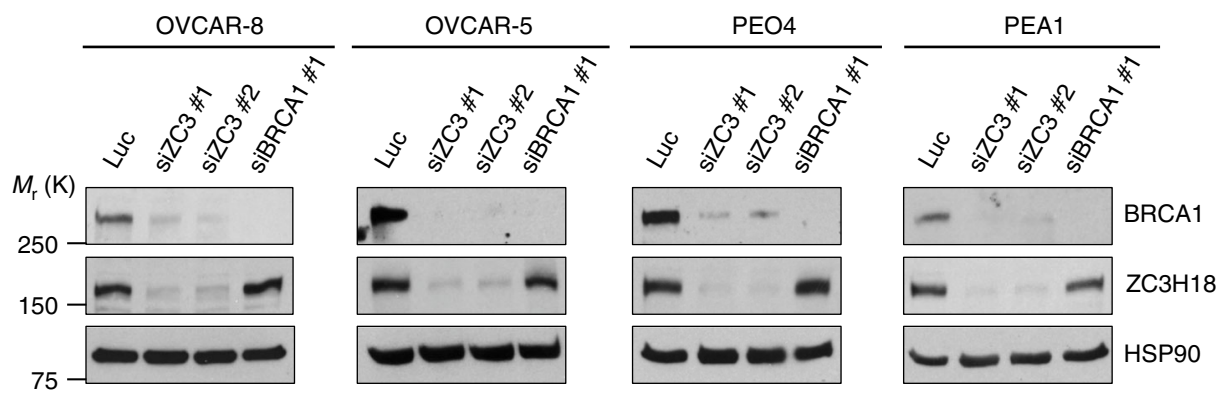

b
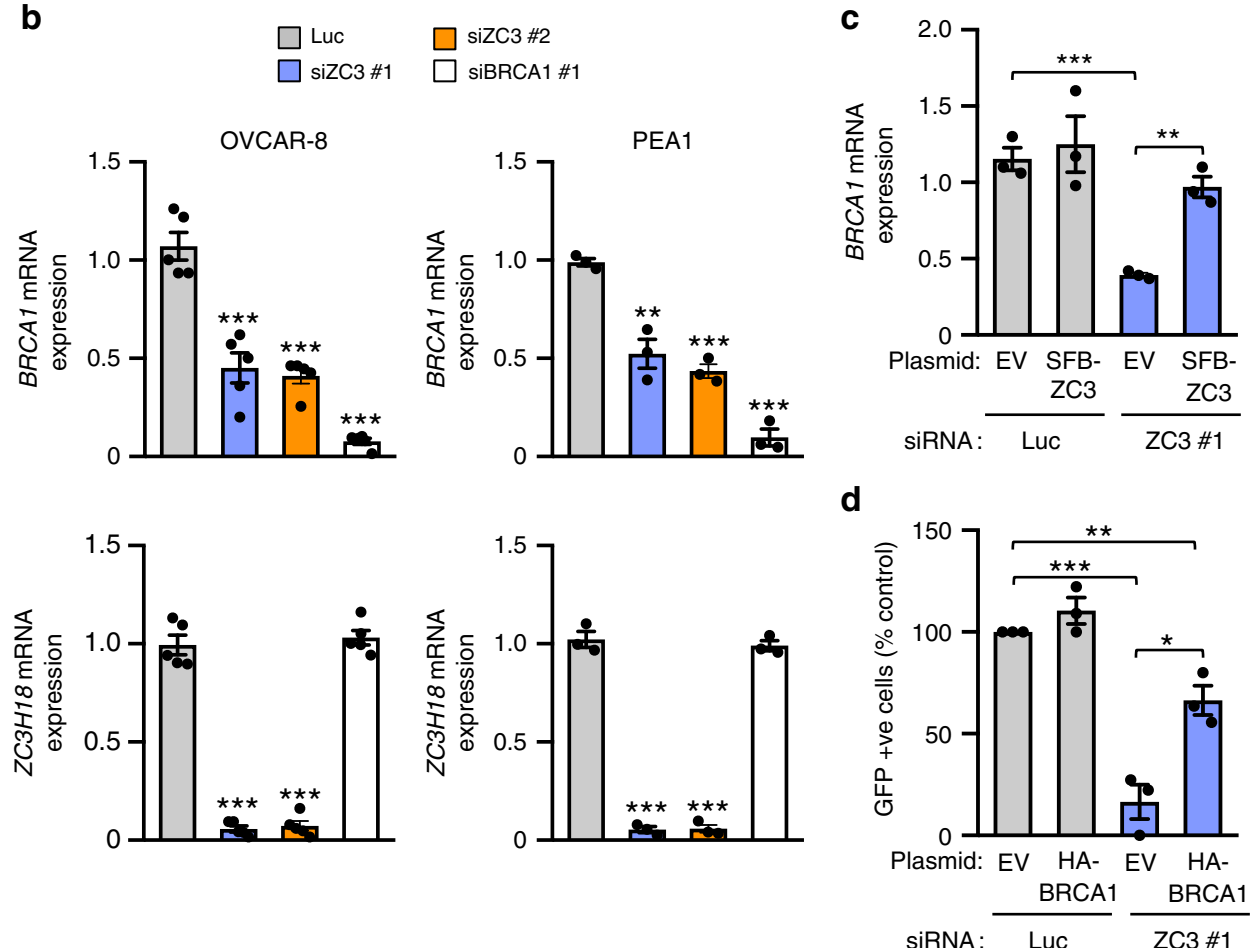

Fig. 2 ZC3H18 depletion reduces BRCA1 mRNA and protein levels. a and $\mathbf{b}$ Indicated cell lines were transfected with control luciferase (Luc), ZC3H18 (ZC3), or BRCA1 siRNAs. Forty-eight hours after transfection, the cells were immunoblotted for the indicated antigens (a) and analyzed by qRT-PCR for ZC3H18 and BRCA1 mRNA levels, which are expressed relative to GAPDH mRNA levels as an internal control (b). c Rescue of BRCA1 mRNA and protein in ZC3H18-depleted OVCAR-8 cells with siRNA-resistant SFB-ZC3H18. OVCAR-8 cells were transfected with Luc or ZC3H18 siRNA plus empty vector (EV) or siRNA-resistant ZC3H18 plasmid (SFB-ZC3H18). BRCA1 mRNA and proteins levels were assessed by qRT-PCR (c) and immunoblotting (Supplementary Fig. 4b), respectively. d Ectopic BRCA1 expression rescues the HR defect caused by ZC3H18 depletion. OCVAR-8 DR-GFP cells were transfected with empty vector (EV) or HA-BRCA1-expressing plasmid with either control luciferase (Luc) or ZC3H18 (ZC3) siRNAs. Seventy-two hours later, the cells were analyzed for GFP by flow cytometry. Representative immunoblots in a are provided from three independent experiments. Unprocessed blots are provided in Source data file. Shown are means \pm SEM from three independent experiments in $\mathbf{b}$-d. ${ }^{\star} p<0.05,{ }^{\star \star} p<0.01$, ${ }^{\star \star \star} p<0.001$, unpaired Student's $t$-test

bidirectional, 250-bp region that drives transcription of BRCA1 and the opposing gene NBR2 (Fig. 6a). Mutation of either site disrupts BRCA1 promoter activity in ovarian cancer cell lines (Supplementary Fig. 9a), demonstrating that both are required for full transcriptional activation of the BRCA1 promoter in these cells. Based on the observation that $\mathrm{ZC} 3 \mathrm{H} 18$ alters the binding of E2Fs to the $B R C A 1$ promoter, we next hypothesized that $\mathrm{ZC} 3 \mathrm{H} 18$ directly binds to one of these E2F sites. To test this idea, we performed electrophoretic mobility shift assays (EMSAs) using bacterially expressed, purified ZC3H18 (Supplementary Fig. 9b). These studies showed that $\mathrm{ZC} 3 \mathrm{H} 18$ directly binds a BRCA1 promoter fragment with wild-type E2FA and E2FB sites $\left(\mathrm{E} 2 \mathrm{FA} / \mathrm{B}^{\mathrm{WT}}\right.$ ) (Fig. 6b). The specificity of the interaction was confirmed by cold probe competition, supershift assay, and random probe competition (Fig. 6b). Analyses using fragments with mutations in the $\mathrm{E} 2 \mathrm{FA}\left(\mathrm{E} 2 \mathrm{~F}^{\triangle \mathrm{A}}\right), \mathrm{E} 2 \mathrm{FB}\left(\mathrm{E} 2 \mathrm{~F}^{\Delta \mathrm{B}}\right)$, or both
$\left(\mathrm{E} 2 \mathrm{~F}^{\mathrm{AA}} / \mathrm{B}\right.$ ) sites (Fig. 6a) showed that $\mathrm{ZC} 3 \mathrm{H} 18$ binds the E2FA site but not the E2FB site (Fig. 6b). In contrast, purified E2F4 binds the E2FB site (Supplementary Fig. 9c, d), whereas purified E2F1 binds to both E2FA and E2FB sites (Supplementary Fig. 9e, f). In agreement with these in vitro observations, we found that E2F4 and $\mathrm{ZC} 3 \mathrm{H} 18$ simultaneously occupy the BRCA1 promoter in cells (Fig. 6c, d) using ChIP-Re-ChIP assays, which can detect the binding of two proteins on a single DNA sequence ${ }^{35}$.

We next investigated how ZC3H18 and E2F4 affect binding of E2F1, which represses BRCA1 transcription, to the BRCA1 promoter. We performed EMSAs using a fixed amount of E2F1 with increasing amounts of ZC3H18 or E2F4. Because ZC3H18 interacts with the $\mathrm{E} 2 \mathrm{FA}$ site, we used the $\mathrm{E} 2 \mathrm{~F}^{\triangle \mathrm{B}}$ promoter probe to test the ability of ZC3H18 to regulate E2F1 binding. Similarly, because E2F4 interacts with the E2FB site, we used the E2F $\triangle \mathrm{A}$ promoter probe to test the ability of E2F4 to regulate E2F1 
a

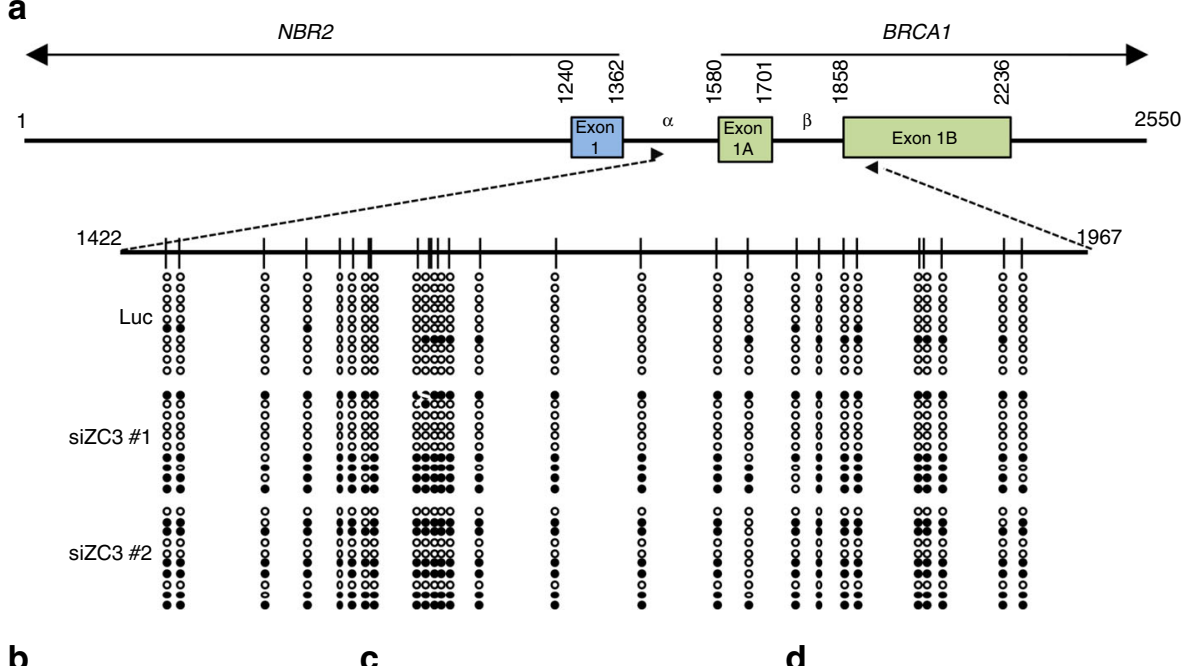

b

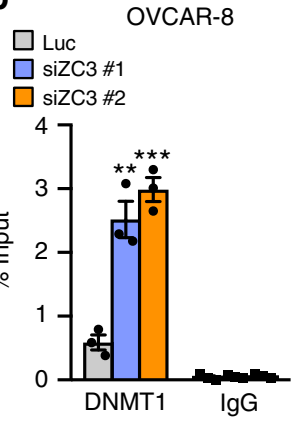

C

OVCAR-8

d
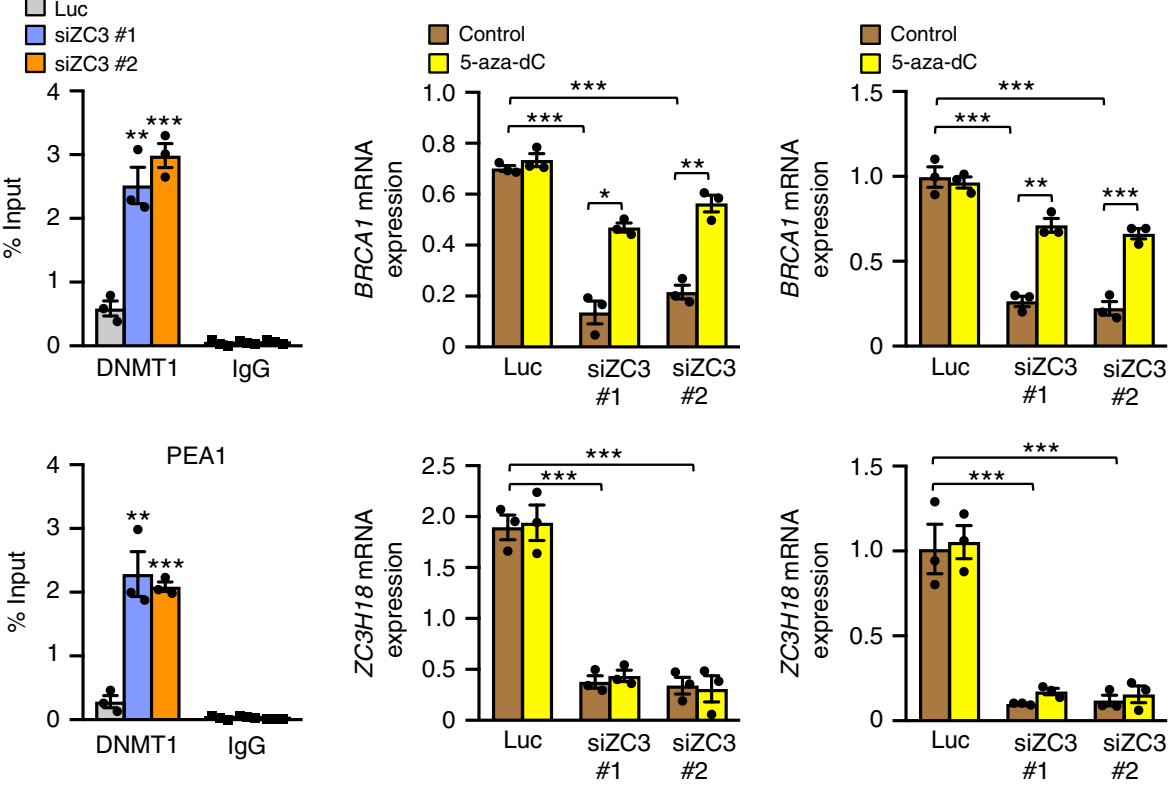

Fig. $3 \mathrm{ZC} 3 \mathrm{H} 18$ depletion causes hypermethylation of the BRCA1 promoter. a Map of the divergent BRCA1/NBR2 promoter with CpG islands indicated by circles below the map. Methylation patterns obtained by bisulfite sequencing of 10 individual clones of PCR products from genomic DNA of control luciferase (Luc) and ZC3H18 siRNA (siZC3)-transfected OVACR-8 cells. Methylated (filled circles) and unmethylated (open circles) CpG positions are shown. b ChIP assays showing increased DNMT1 occupancy on the BRCA1 promoter in ZC3H18-depleted cells. OVCAR-8 (top panel) and PEA1 (bottom panel) cells transfected with control luciferase (LuC) or ZC3H18 siRNAs were harvested $48 \mathrm{~h}$ after transfection and processed for ChIP to detect DNMT1 on the BRCA1 promoter. c and d OVCAR-8 (c) and PEA1 (d) cells were transfected with control (Luc) or ZC3H18 siRNAs and treated with vehicle or 5-aza-2'deoxycytidine $(5 \mu \mathrm{M})$ for 3 days. BRCA1 mRNA (top panel) and ZC3H18 mRNA (bottom panel) levels were analyzed by qRT-PCR. The mRNA levels are normalized to GAPDH mRNA levels as the internal control. Data are means \pm SEM from three independent experiments in $\mathbf{b}-\mathbf{d}$. ${ }^{\star} p<0.05,{ }^{* \star} p<0.01,{ }^{\star \star *} p<$ 0.001 , unpaired Student's $t$-test

binding. Both ZC3H18 and E2F4 abolished E2F1 interaction with both E2F binding sites (Fig. 6e, f). These results suggest that ZC3H18 binding to the E2FA site - and E2F4 binding to the $\mathrm{E} 2 \mathrm{FB}$ site - prevents the interaction of E2F1 with the BRCA1 promoter, thereby blocking E2F1-mediated repression of BRCA1.

ZC3H18 and E2F4 levels correlate with BRCA1 levels in HGSOC. Our mechanistic studies discovered that $\mathrm{ZC} 3 \mathrm{H} 18$ and E2F4 depletion reduces BRCA1 levels in ovarian cancer cell lines and low-passage, short-term ex vivo-cultured HGSOC PDX models freshly isolated from mice (Figs. 2a, b, 5b, c). To further address whether ZC3H18 and E2F4 affect BRCA1 expression in HGSOC, we compared BRCA1 mRNA levels with ZC $3 H 18$ and E2F4 mRNA levels in HGSOC tumors from patients and from
PDX mouse models. This analysis showed that BRCA1 mRNA levels were positively correlated with both $\mathrm{ZC} 3 \mathrm{H} 18$ and E2F4 mRNA levels in patients (ZC3H18: $r=0.19, p=0.057$; E2F4: $r=$ 0.37, $p<0.001)$ and PDX models (ZC3H18: $r=0.33, p<0.001$; E2F4: $r=0.34, p<0.001$ ) (Fig. 7a; Supplementary Data 2). Taken together, these findings suggest that $\mathrm{ZC} 3 \mathrm{H} 18$ and $\mathrm{E} 2 \mathrm{~F} 4$ play a role in regulating BRCA1 expression in HGSOCs.

\section{Discussion}

As summarized in Fig. $7 \mathrm{~b}$, we found that $\mathrm{ZC} 3 \mathrm{H} 18$ is a DNA binding protein that regulates $B R C A 1$ transcription by directly interacting with the E2FA site in the BRCA1 promoter. $\mathrm{ZC} 3 \mathrm{H} 18$ binding to the E2FA site promotes E2F4 interaction with the adjacent E2FB. Co-occupancy of ZC3H18 and E2F4 on adjacent 
a

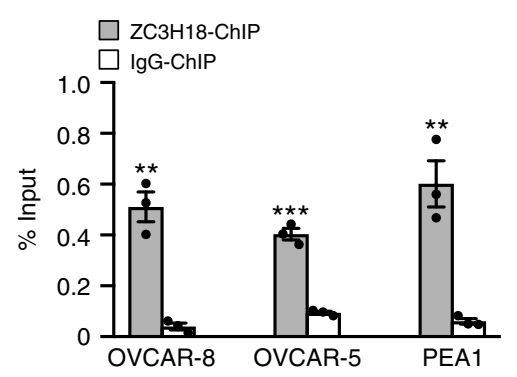

d
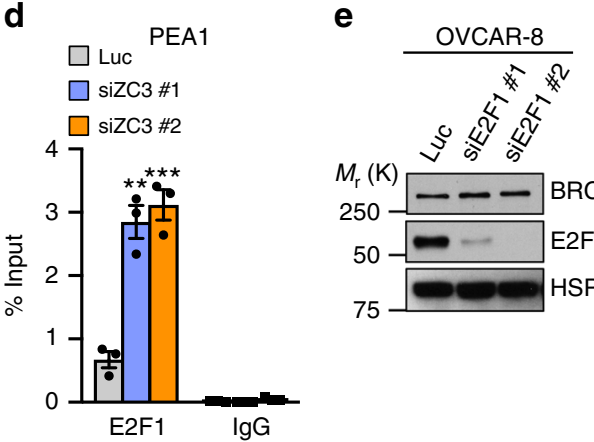

b

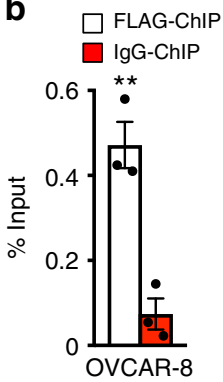

c

OVCAR-8

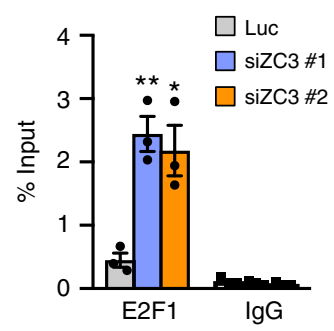

f

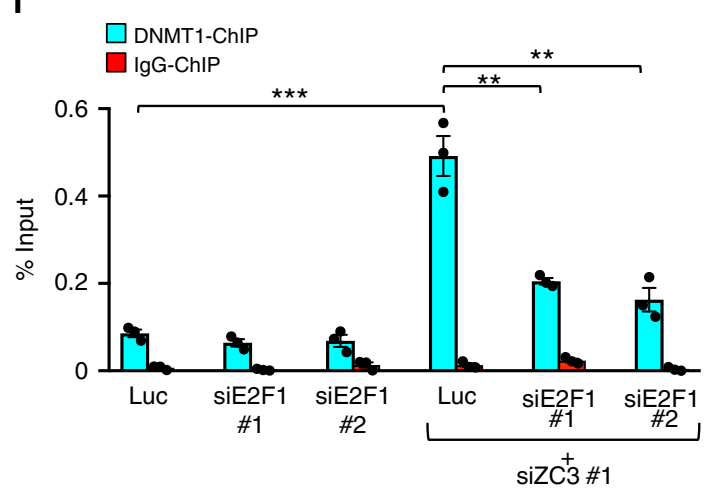

g

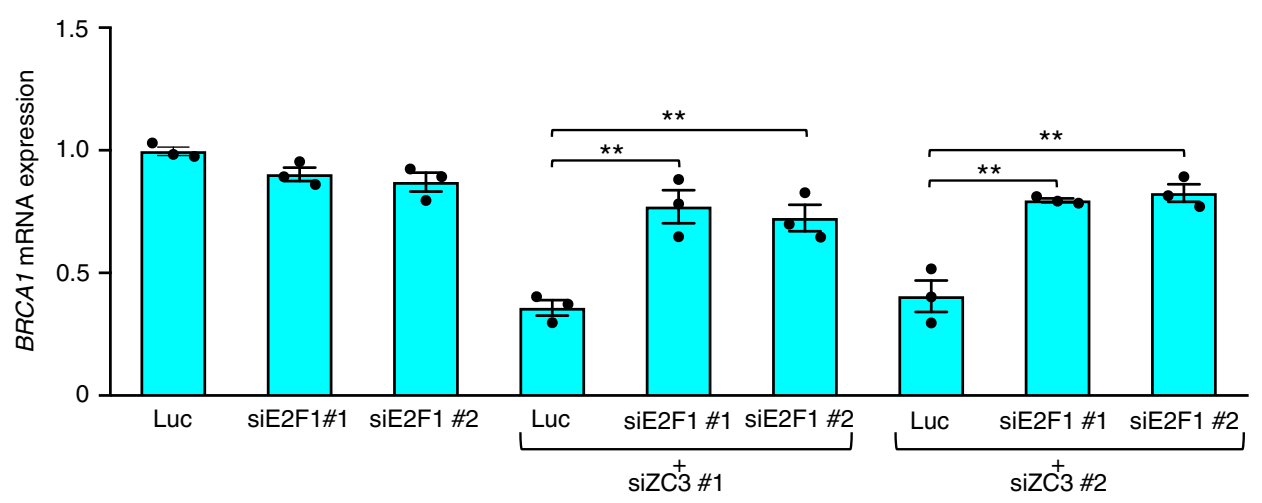

Fig. 4 ZC3H18 occupies the BRCA1 promoter and regulates E2F1. a OVCAR-8, OVCAR-5, and PEA1 cells were processed for ChIP assays using anti-ZC3H18 or IgG control antibodies and primers specific for the BRCA1 promoter. b OVCAR-8 cells transiently expressing SFB-ZC3H18 were processed for ChIP assays using FLAG or IgG control antibodies. c, d ChIP assays showing increased occupancy of E2F1 on the BRCA1 promoter when ZC3H18 is depleted. OVCAR-8 (c) and PEA1 (d) cells transfected with control luciferase (Luc) or ZC3H18 siRNAs (siZC3) were processed for ChIP using anti-E2F1 or IgG control antibodies $48 \mathrm{~h}$ after transfection. e E2F1 depletion does not affect BRCA1 levels. OVCAR-8 cells were transfected with control luciferase (Luc) or two independent E2F1 siRNAs and analyzed for BRCA1, E2F1, and HSP90 levels by immunoblotting $48 \mathrm{~h}$ later. $\mathbf{f}$ DNMT1 occupancy on the BRCA1 promoter requires E2F1. OVCAR-8 cells transfected with Luc, E2F1, or ZC3H18 siRNA with our without E2F1 siRNAs were processed for ChIP with DNMT1 or control IgG antibodies $48 \mathrm{~h}$ after transfection. $\mathrm{g}$ Depletion of E2F1 restores BRCA1 expression when ZC3H18 is depleted. OVCAR-8 cells transfected with Luc, E2F1, or $\mathrm{ZC} 3 \mathrm{H} 18$ siRNAs with our without E2F1 siRNAs were analyzed for BRCA1 mRNA levels by qRT-PCR. BRCA1 mRNA levels were normalized to GAPDH mRNA levels. Data in $\mathbf{a}-\mathbf{g}$ are means \pm SEM from three independent experiments. ${ }^{\star \star} p<0.01,{ }^{\star \star \star} p<0.001$, unpaired Student's $t$-test. Representative immunoblots in e are provided from three independent experiments. Unprocessed blots are provided in Source data file

E2F sites prevents E2F1 binding to either E2F site and promotes $B R C A 1$ transcription. In contrast, when $\mathrm{ZC} 3 \mathrm{H} 18$ is depleted, the E2FA and E2FB sites are instead occupied by E2F1, which represses BRCA1 by recruiting DNMT1 and causing CpG hypermethylation at sites previously found to reduce BRCA1 expression in patients $6,27,36,37$. In agreement with these observations, loss of $\mathrm{ZC} 3 \mathrm{H} 18$ reduces BRCA1 levels, disrupts $\mathrm{HR}$, and sensitizes ovarian cancer cells to the DNA crosslinking agents, cisplatin and melphalan, as well as the PARP inhibitors veliparib and olaparib.

The E2F family of transcription factors consists of eight genes (E2F1-8) that encode nine different proteins ${ }^{38}$. E2F1, E2F2, and E2F3A can activate or repress transcription depending on whether they interact with pocket proteins, such as retinoblastoma (Rb), p107, and p130 that recruit DNMTs and other enzymes that silence target genes ${ }^{32,34}$. In contrast, E2F3B, E2F4, E2F5, E2F6, E2F7, and E2F8 were originally classified as repressors $^{38}$; however, additional studies have shown that E2F4 also activates transcription of multiple genes ${ }^{29,39}$. The roles of E2F family members in BRCA1 regulation have been primarily investigated in breast cancer cell lines ${ }^{15,30,32,33}$. Consistent with the typically accepted roles of E2F1 and E2F4, these studies have generally concluded that E2F1 activates BRCA1, whereas E2F4 represses $B R C A 1$ transcription. In contrast, our studies in multiple ovarian cancer cell lines found that E2F1 represses BRCA1 expression, and that this repression requires the DNA 


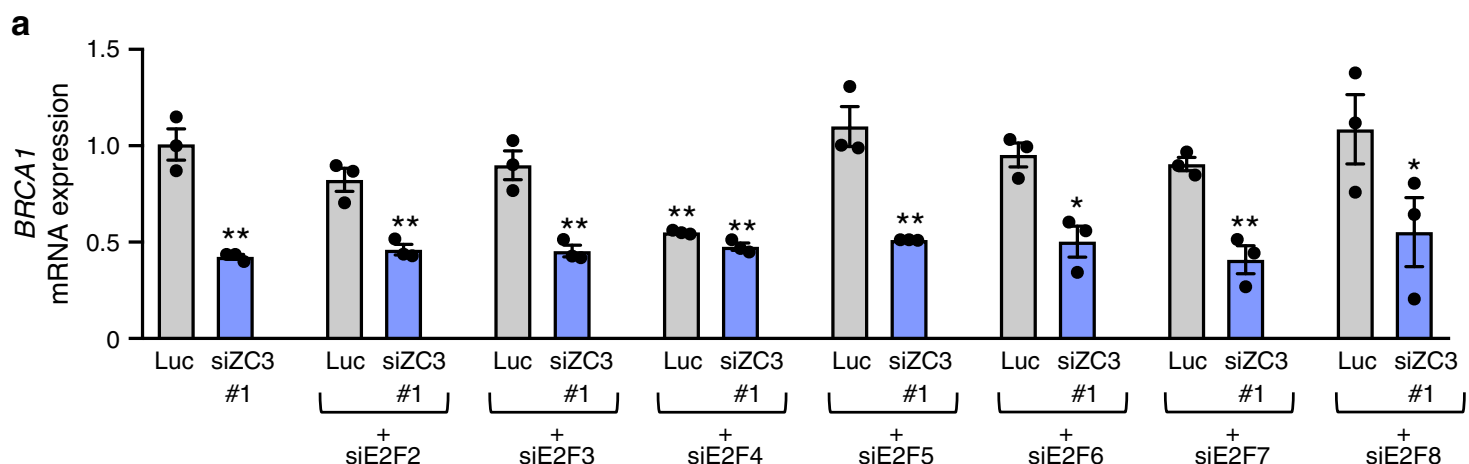

b

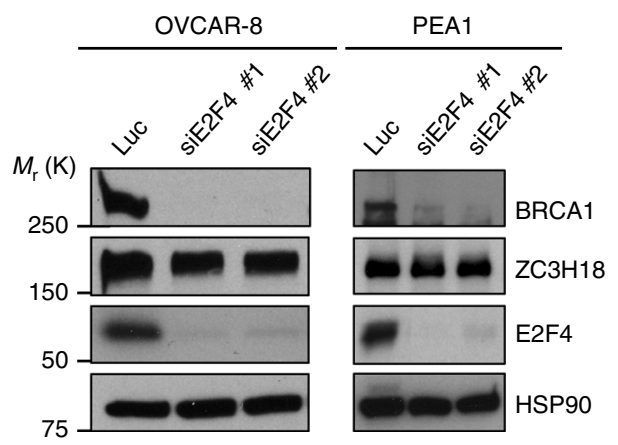

C

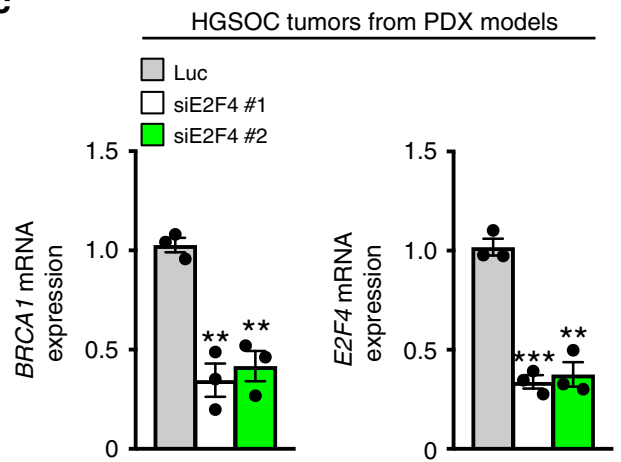

d

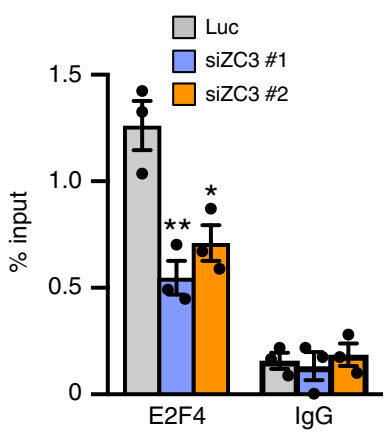

e

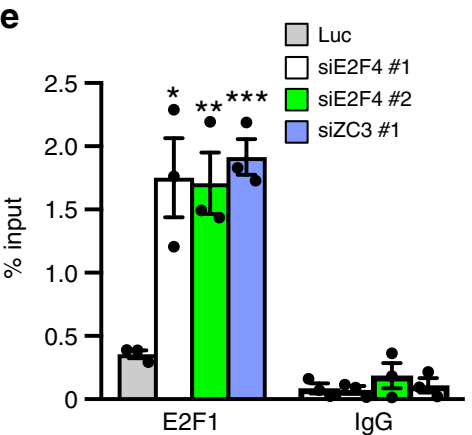

f

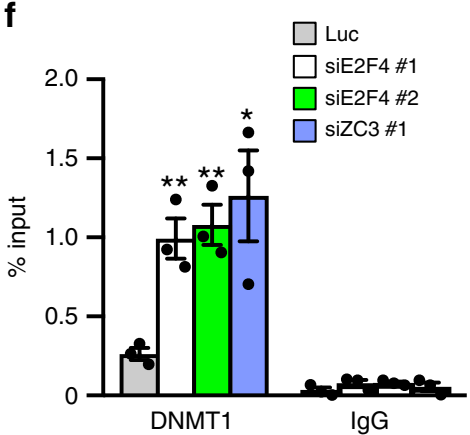

g

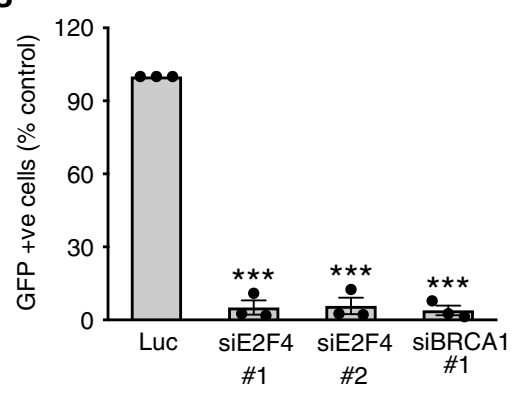

h

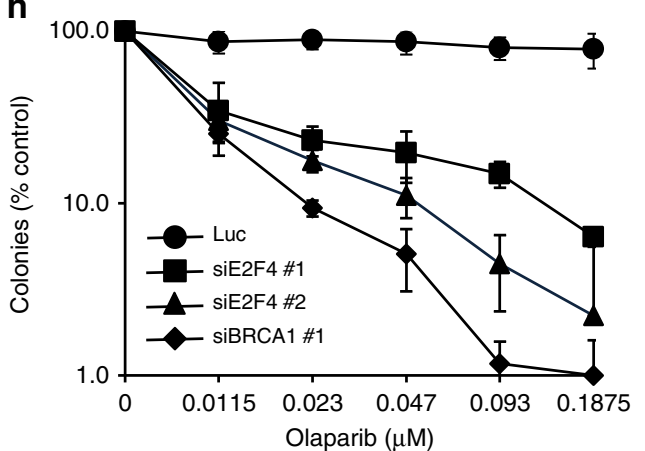

methyltransferase DNMT1 and is correlated with CpG hypermethylation of the BRCA1 promoter.

We also found unexpected results with E2F4. Using an siRNA screen, we found that (1) of all the E2F family members, only E2F4 depletion reduced BRCA1 expression in OVCAR-8 cells; (2) $B R C A 1$ expression in multiple ovarian cancer cell lines was reduced by two independent E2F4 siRNAs; (3) E2F4 depletion in short-term ex vivo cultures of freshly isolated HGSOC tumors from mouse PDX models reduced BRCA1 expression; and (4)
E2F4 expression is positively correlated with BRCA1 expression in primary HGSOCs and ovarian cancer PDXs. Suprisingly, however, E2F4 was shown to repress BRCA1 expression in breast cancer and other cell lines ${ }^{32}$. While we do not currently understand the underlying mechanism for this alternative regulation, $\mathrm{E} 2 \mathrm{~F} 4$ is converted into an activator when $\mathrm{Rb}$ family members are lost ${ }^{40}$. Accordingly, we speculate that differential expression of $\mathrm{Rb}$ family members and/or posttranslational modifications of $\mathrm{Rb}$ that regulate interactions with E2F family members may contribute to 
Fig. $5 \mathrm{ZC} 3 \mathrm{H} 18$ promotes binding of E2F4 and activation of the BRCA1 promoter. a qRT-PCR analysis of BRCA1 mRNA expression. OVCAR-8 cells transfected with control luciferase (Luc), E2F2, E2F3, E2F4, E2F5, E2F6, E2F7, or E2F8 siRNAs with or without ZC3H18 siRNA were analyzed by qRT-PCR for BRCA1 mRNA levels, which were normalized to GAPDH mRNA. b Immunoblots of indicated proteins in OVCAR-8 and PEA1 cells transfected with control luciferase (Luc) and two independent E2F4 siRNAs. c BRCA1 mRNA expression, normalized to GAPDH mRNA, was determined by qRT-PCR in short-term ex vivo cultures of HGSOC tissues from PDX models electroporated with Luc or E2F4 siRNAs. d ZC3H18 promotes E2F4 occupancy on the BRCA1 promoter. OVCAR-8 cells transfected with control luciferase (Luc) or ZC3H18 siRNAs were analyzed by ChIP for E2F4 bound to the BRCA1 promoter. e, $\mathbf{f}$ Depletion of E2F4 or ZC3H18 promotes E2F1 and DNMT1 occupancy on the BRCA1 promoter. OVCAR-8 cells transfected with Luc, E2F4, and ZC3H18 siRNAs were analyzed by ChIP for E2F1 (e) and DNMT1 (f) accumulation on the BRCA1 promoter. $\mathbf{g}$ E2F4 depletion disrupts HR. OVCAR-8-DRGFP cells transfected with $p C \beta A S c e l$ plus indicated siRNAs were analyzed for GFP fluorescence by flow microfluorimitry $48 \mathrm{~h}$ after transfections. HR efficiencies were normalized to control (Luc) siRNA-transfected cells. h OVCAR-8 cells were transfected with control luciferase (Luc), E2F4, or BRCA1 siRNAs. Forty-eight hours later, the cells were trypsinized, re-plated, and allowed to adhere for $24 \mathrm{~h}$. The indicated concentrations of olaparib were then added, and the cells were cultured for 10 days, stained with Coomassie Blue, and colonies were counted manually. Data are means \pm SEM of three independent experiments. ${ }^{\star} p<0.05,{ }^{\star \star} p<0.01,{ }^{\star \star \star} p<0.001$, unpaired Student's $t$-test. Representative immunoblots in $\mathbf{b}$ are provided from three independent experiments. Unprocessed blots are provided in Source data file. The graph in $\mathbf{h}$ represents one of three independent experiments that gave similar results. Error bars are standard deviation of triplicate wells from a representative experiment

the disparate regulation of $B R C A 1$ in ovarian versus breast cancer. Taken together these results suggest that E2F4 is an activator of BRCA1 transcription in ovarian cancer cells.

Notably, the present studies also uncovered a mechanism by which $\mathrm{ZC} 3 \mathrm{H} 18$ regulates gene expression, namely that $\mathrm{ZC} 3 \mathrm{H} 18$ is a DNA binding protein that interacts with a specific site in the $B R C A 1$ promoter. These findings add to the complex array of functions already ascribed to $\mathrm{ZC} 3 \mathrm{H} 18$. These include activating the transcription factor NF- $\kappa B$ via an unknown mechanism ${ }^{41}$, regulating RNA metabolism by participating in mRNA splicing and export from the nucleus ${ }^{18}$, and targeting RNA for exosomemediated degradation ${ }^{19}$. However, because (1) ZC3H18 depletion did not affect $B R C A 1 \mathrm{mRNA}$ splicing or stability (Supplementary Fig. 5a, b) and (2) ZCCHC8 depletion, which disrupts the CBCN complex, did not affect BRCA1 levels (Supplementary Fig. 5c), it is unlikely that $\mathrm{ZC} 3 \mathrm{H} 18$ is regulating BRCA1 expression by altering the metabolism of BRCA1 RNAs. Instead, our results demonstrate that ZC3H18 directly binds DNA to activate BRCA1 transcription. Consistent with our findings, while this manuscript was in preparation, Winczura et al. ${ }^{17}$ reported that $\mathrm{ZC} 3 \mathrm{H} 18$ depletion reduced the transcription of a subset of genes, including BRCA1. Using ChIP assays, they also showed that $\mathrm{ZC} 3 \mathrm{H} 18$ associates with the $B R C A 1$ promoter; however, the mechanism by which $\mathrm{ZC} 3 \mathrm{H} 18$ increased $B R C A 1$ expression was not identified. Here we have identified a key mechanism by which $\mathrm{ZC} 3 \mathrm{H} 18$ regulates the $B R C A 1$ promoter by showing that $\mathrm{ZC} 3 \mathrm{H} 18$ 's ability to bind directly to the $B R C A 1$ promoter and regulate the association of E2F family members is a major driver of BRCA1 expression in ovarian cancer. However, as shown in Fig. 2a, b, $\mathrm{ZC} 3 \mathrm{H} 18$ has a greater effect on BRCA1 protein levels than on its mRNA levels, suggesting that $\mathrm{ZC} 3 \mathrm{H} 18$ may also regulate BRCA1 posttranscriptionally.

The findings presented here raise the possibility that $\mathrm{ZC} 3 \mathrm{H} 18$ loss contributes to HR defects by reducing BRCA1 expression. Consistent with this possibility, deep ZC3H18 deletions and low ZC3H18 mRNA levels are nearly mutually exclusive with BRCA1 driver mutations and deep deletions in ovarian tumors analyzed by The Cancer Genome Atlas research network (Supplementary Fig. 10). This correlation was also observed in breast cancer (Supplementary Fig. 10), consistent with our observations that $\mathrm{ZC} 3 \mathrm{H} 18$ regulates $B R C A 1$ in breast cancer cells. Accordingly, our findings suggest that loss and/or decreased ZC3H18 expression may help identify HGSOC patients most likely to benefit from PARP inhibitor and platinum-based therapies.

\footnotetext{
Methods

Cell lines, cell culture, and small molecules. The OVCAR- 8 and OVCAR- 5 cells were kind gifts from D. Scudierio (NCI, National Institutes of Health). OVCAR-8-
}

DR-GFP cells, which have a genomically integrated DR-GFP substrate for HR repair assays, were described previously ${ }^{42}$. The PEA1 and PEO4 cells were from Sigma-Aldrich and Dr. T. Taniguchi (Fred Hutchinson Cancer Research Center), respectively. The cells were cultured in RPMI-1640 medium (Corning) supplemented with $8 \%$ fetal bovine serum (Millipore). All cells were authenticated by autosomal STR profiling (University of Arizona Genetics Core) and maintained in a humidified $37^{\circ} \mathrm{C}$ incubator with $5 \% \mathrm{CO}_{2}$. Cisplatin was obtained from Teva Pharmaceuticals. Melphalan, veliparib (ABT-888), and olaparib (AZD2281) were from Selleck Chemicals.

siRNA transfections. siRNAs were purchased from Dharmacon. siRNA transfections $(2 \mu \mathrm{M} /$ transfection) were performed using a BTX ECM 830 electroporator Cells $\left(5 \times 10^{6} /\right.$ transfection) were mixed with $20 \mu \mathrm{L}$ of $20 \mu \mathrm{M}$ siRNA solution in a 4-mm electroporation cuvette in $200 \mu \mathrm{L}$ RPMI supplemented with $8 \%$ fetal bovine serum and electroporated with two $10-\mathrm{mS}$ pulses at $280 \mathrm{~V}$ as previously described $^{42}$. siRNAs used were:

luciferase, 5'-CUUACGCUGAGUACUUCGA-3';

BRCA1, 5'-GUGGGUGUUGGACAGUGUA-3';

E2F1 \#1, 5'-UCGGAGAACUUUCAGAUCU-3';

E2F1 \#2, 5'-GAGAAGUCACGCUAUGAGA-3';

E2F4 \#1, 5'-GAGAUACCCUCUUGGCCAU-3';

E2F4 \#2, 5'-CAGAAGAAGUACCAGAUUC-3';

ZC3H18 \#1, 5'-GAAGCGCUAUGAACCAUCA-3';

ZC3H18 \#2, 5'-GAACGAGGACUCCGGCAUG-3'

siRNAs used in Supplementary Fig. S1A were siGENOME SMARTpool siRNAs (Dharmacon) that contain a pool of four different siRNAs that target various regions of each gene.

Plasmids and transfections. Human ZC3H18 cDNA (Dharmacon, MHS6278202759301), E2F4 cDNA (Addgene plasmid \#10914) ${ }^{43}$, and E2F1 cDNA (Addgene plasmid $\# 24225)^{44}$ were subcloned into the pSFB vector that contains in-frame Nterminal S-peptide, FLAG, and streptavidin-binding peptide tags ${ }^{45}$. Luciferase reporter assays used $\mathrm{pBRC}-\mathrm{FF}$, which contains the $B R C A 1$ promoter driving firefly luciferase expression (a kind gift from Dr. Peter Glazer, Yale University) ${ }^{15}$. To introduce E2FA and/or E2FB site mutations into pBRC-FF, PCR-based sitedirected mutagenesis was performed using the following: for E2FA site mutation 5'-CGGTAGCCCCTTGGCACACGTGGCAACGGAAAAG-3' (sense) and 5'-CT TTTCCGTTGCCACGTGTGCCAAGGGGCTACCG-3' (antisense); for E2FB site mutation, 5'-CCGTGGCAACGGAAAAGCGCGTACGTTACAGATAAATTAA AACTG-3' (sense) and 5'-CAGTTTTAATTTATCTGTAACGTACGCGCTTT TCCGTTGCCACGG-3' (antisense). The pRL-SV40 Renilla luciferase reporter construct was from Promega. Transfection of plasmids was as described ${ }^{42}$. For $E$. coli expression and purification of human ZC3H18, N-terminally SFB-tagged $\mathrm{ZC} 3 \mathrm{H} 18$ was subcloned into the $\mathrm{pET}-24 \mathrm{a}(+)$ expression vector with an in-frame C-terminal 6X His-Tag (Novagen). An HA-tagged human full-length BRCA1 plasmid construct was described before ${ }^{46}$. The pCßASceI plasmid was obtained from Addgene (Addgene plasmid \#26477) ${ }^{47}$. All plasmid constructs generated were confirmed by Sanger sequencing.

Plasmid transfections were performed as described for siRNA transfections above except that transfections used $1-1.5 \times 10^{7}$ cells and $40 \mu \mathrm{g}$ of plasmid (using a combination of empty vector and gene of interest to obtain $40 \mu \mathrm{g}$ ) per transfection.

MTS and clonogenic assays. Twenty-four hours after siRNA transfection, cells were seeded in 96-well plates $\left(1.2 \times 10^{5}\right.$ cells/well $)$ and incubated for additional 24 h. The cells were then treated with the DNA crosslinking agents, cisplatin, or melphalan, for 3 days or PARP inhibitors, veliparib or olaparib, for 7 days. Cell viability following drug exposure was detected using MTS [(3-(4,5-dimethylthiazol2-yl)-5-(3-carboxymethoxyphenyl)-2-(4-sulfophenyl)-2H-tetrazolium)/phenazine 


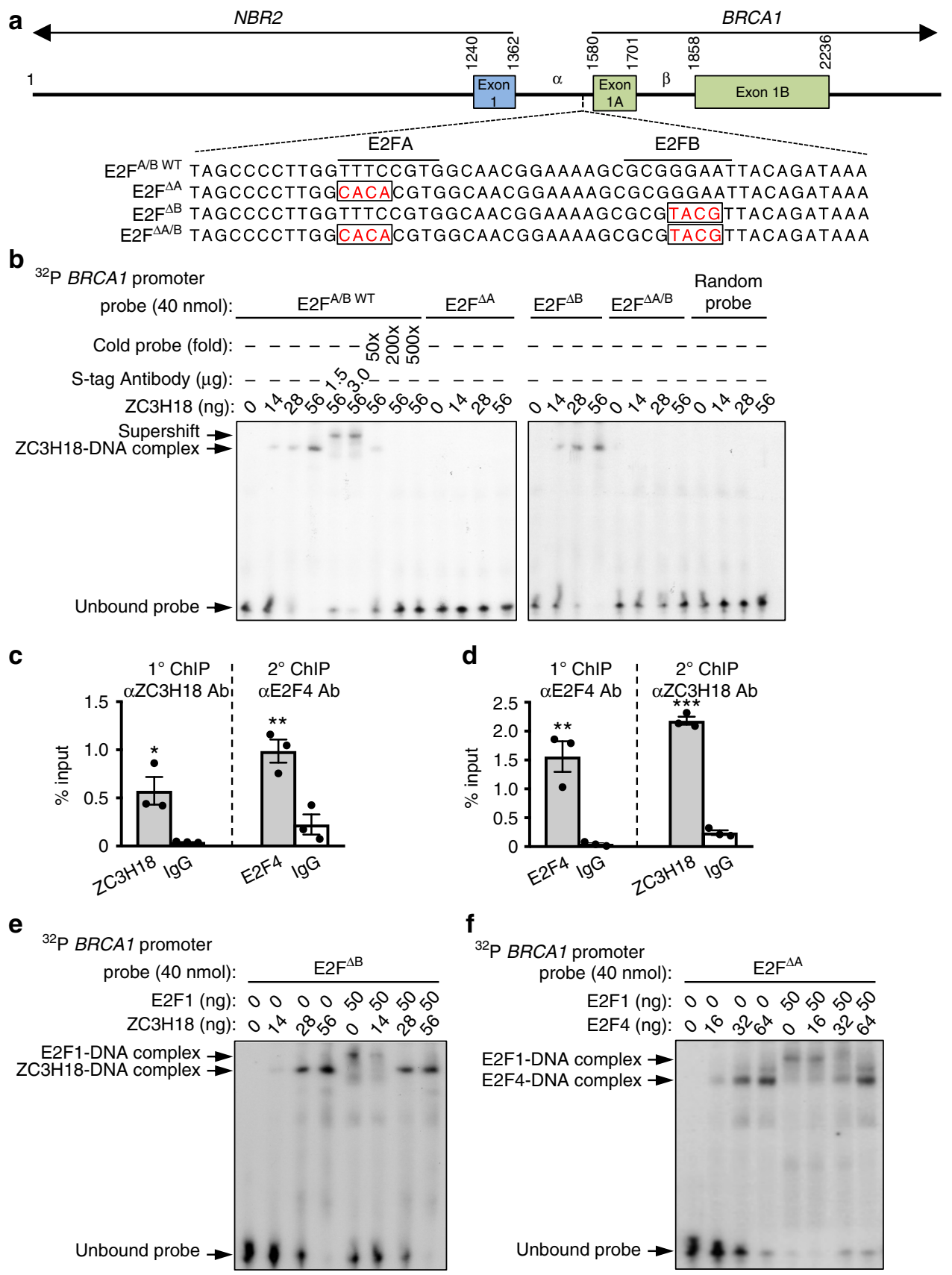

Fig. $6 \mathrm{ZC} 3 \mathrm{H} 18$ binds the BRCA1 promoter and inhibits E2F1 binding. a Schematic of the BRCA1 proximal promoter with E2FA and E2FB sites indicated. Nucleotide sequences of the DNA probes used in the electrophoretic mobility shift assays (EMSA). E2FA and E2FB mutation sites are indicated in open rectangles. b EMSA with purified recombinant SFB-ZC3H18 using BRCA1 promoter probe with wild-type sequence (E2FA/BWT) or mutations in the E2FA site $(E 2 F \Delta A)$, the $E 2 F B$ site $(E 2 F \Delta B)$, or both $E 2 F$ sites $(E 2 F \triangle A / B)$. A probe with randomly shuffled sequences was used as negative control. For supershift assays, an anti-S-Tag monoclonal antibody, which binds the SFB tag in SFB-ZC3H18, was used. c, d ZC3H18 and E2F4 co-occupy the endogenous BRCA1 promoter. Sequential ChiP (ChIP-Re-ChIP) assays in OVCAR-8 cells using anti-ZC3H18 antibody for primary ChIP and anti-E2F4 antibody for secondary ChIP (c) and using anti-E2F4 antibody for primary ChIP and anti-ZC3H18 antibody for secondary ChIP (d). e EMSA with purified recombinant SFB-ZC3H18 and SFB-E2F1 using BRCA1 promoter probe with mutated E2FB site $\left(\mathrm{E} 2 \mathrm{~F}^{\mathrm{B}}\right)$ ). $\mathbf{f} \mathrm{EMSA}$ with purified SFB-E2F4 and SFB-E2F1 using BRCA1 promoter probe with mutated E2FA site $\left(E 2 F^{\triangle A}\right)$. The images of EMSA in $\mathbf{b}, \mathbf{e}$, and $\mathbf{f}$ are representative of three independent experiments that gave similar results. Data in $\mathbf{c}$ and d are means \pm SEM of three independent experiments. ${ }^{*} p<0.05,{ }^{\star \star} p<0.01,{ }^{\star \star *} p<0.001$, unpaired Student's $t$-test

methosulfate (PMS)] colorimetric assay (Promega) according to the supplier's protocol. For clonogenic assays, $48 \mathrm{~h}$ after siRNA transfection, OVCAR- 8 cells were seeded in 6-well plates at 300 cells/well (in triplicate per assay point) and allowed to adhere overnight. The cells were then treated with the indicated concentrations of olaparib, and cultured in the presence of the drug for 8-10 days. Colonies were stained with Coomassie Blue, and colonies of $>50$ cells were counted manually. Inhibition of colony formation was presented as percentage of colonies formed compared to corresponding untreated control.
Cell cycle analysis. Forty-eight hours after siRNA transfection, cells were harvested, fixed with ethanol, and cell cycle analysis was conducted by flow cytometry following staining of the DNA with propidium iodide.

Immunocytochemistry. Forty-eight hours after siRNA transfection, cells were harvested and plated onto 8-well chamber slides (ThermoFisher Scientific), and the cells were allowed to attach for another $24 \mathrm{~h}$. The cells were then irradiated with 2 
a
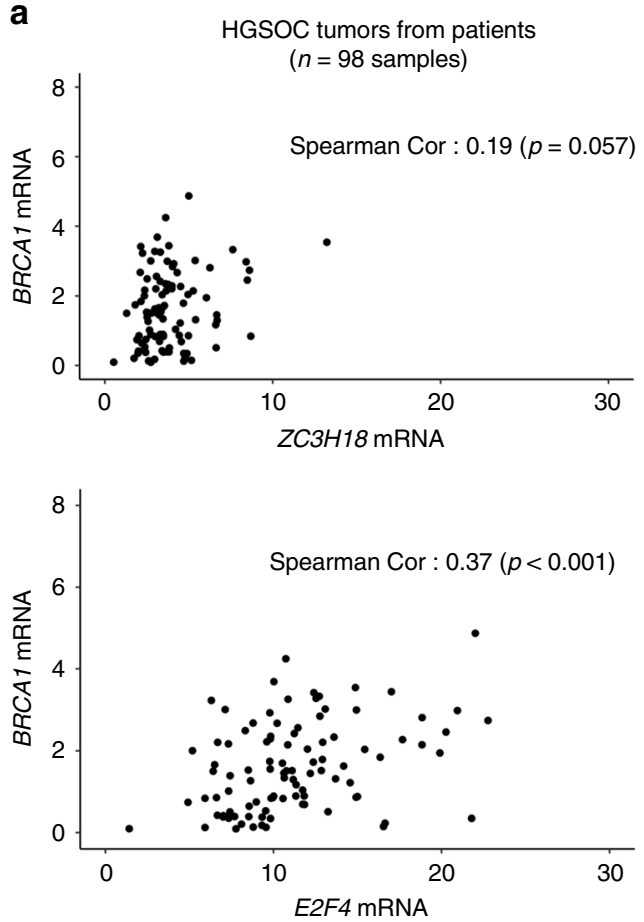

b

\section{ZC3H18-proficient ovarian} cancer cells

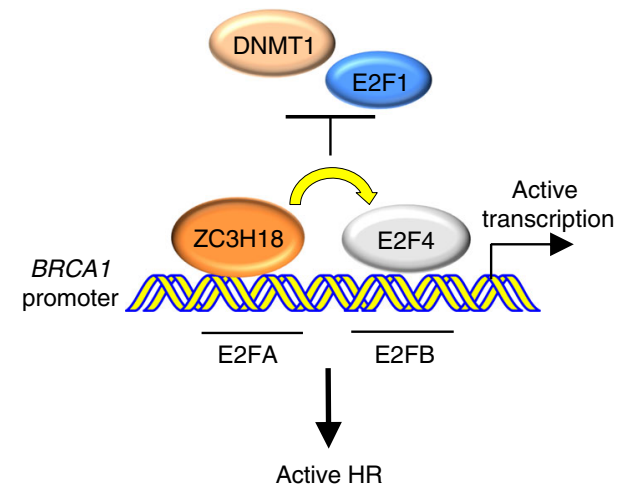

HGSOC tumors from PDX models $(n=130$ samples $)$
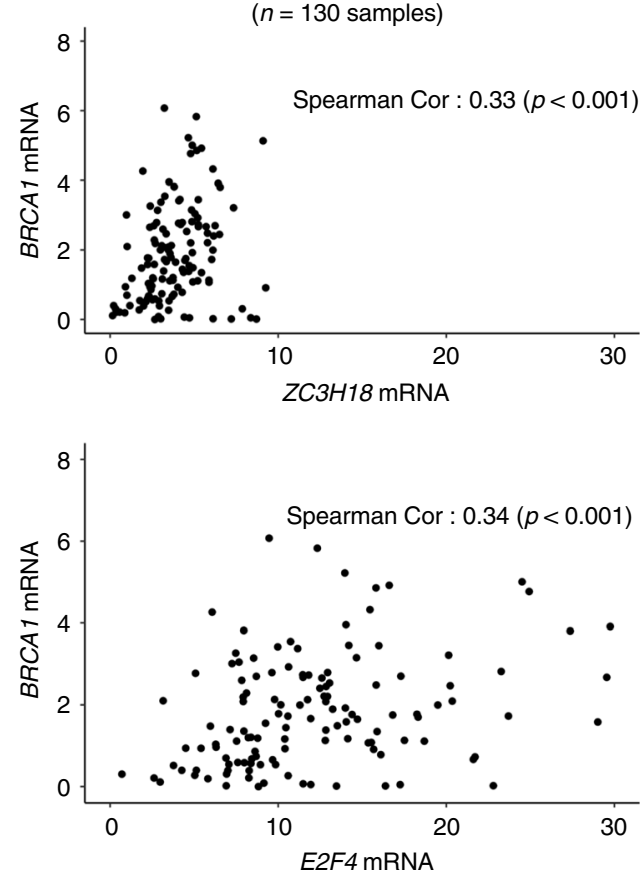

ZC3H18-deficient ovarian cancer cells

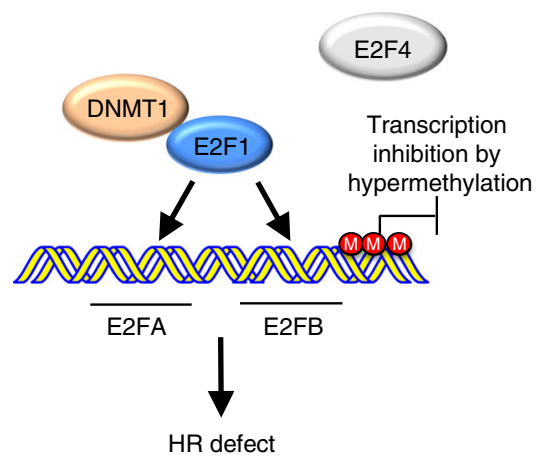

Fig. $7 \mathrm{ZC} 3 \mathrm{H} 18$ and E2F4 expression correlates with BRCA1 levels in HGSOC patient and PDX tumors. a Scatter plots of BRCA1 mRNA expression as a function of either ZC3H18 or E2F4 mRNA expression in HGSOC tumors from patients and PDX models. mRNA expression is in RPKM units. $\mathbf{b}$ Model for the role of $\mathrm{ZC} 3 \mathrm{H} 18$ in $B R C A 1$ transcription. Left panel: in $\mathrm{ZC} 3 \mathrm{H} 18$-proficient cells, $\mathrm{ZC} 3 \mathrm{H} 18$ directly binds to the E2FA site on the BRCA1 promoter, where it promotes E2F4 occupancy at the E2FB site, thereby preventing E2F1-dependent DNMT1 occupancy and promoter methylation and inducing BRCA1 transcription. Right panel: in ZC3H18-deficient cells, E2F1 occupies both E2FA and E2FB sites and causes DNMT1 loading onto the promoter, leading to methylation of the promoter, reduced expression of BRCA1, and disruption of HR. Spearman correlations are shown in the images

Gray of ionizing radiation, incubated at $37^{\circ} \mathrm{C}$ for $6 \mathrm{~h}$, fixed with $3 \%$ paraformaldehyde in phosphate buffered saline (PBS) for $12 \mathrm{~min}$, and permeabilized in $0.25 \%$ Triton X-100 in PBS for 10 min. The slides were blocked with 3\% bovine serum albumin in PBS containing $0.25 \%$ Triton X-100, incubated at room temperature overnight with rabbit polyclonal primary antibody to RAD51 (1:250, PC-130, Calbiochem) and mouse monoclonal antibody to phospho-histone H2A.X $(\gamma$-H2A.X) (1:250, 05-636, Millipore), washed three times with PBS, incubated with anti-rabbit Alexa Fluor ${ }^{\oplus} 488$ and anti-mouse Alexa Fluor ${ }^{\circledR} 594$-conjugated secondary antibodies (1:500; ThermoFisher Scientific) for $1 \mathrm{~h}$ in the dark, washed once with PBS, incubated with Hoechst-33342 (1:1000, ThermoFisher Scientific) for 2 min to stain the nuclei, mounted in Prolong Gold Antifade (ThermoFisher Scientific), and examined with a confocal laser scanning microscope using a $\times 40$ or $\times 100$ objective.

Immunoblotting. Two days after siRNA transfection, cells were harvested and lysed in $50 \mathrm{mM}$ HEPES (pH 7.6), $150 \mathrm{mM} \mathrm{NaCl}, 1 \mathrm{mM}$ EDTA, $1 \%$ Triton X-100, $10 \mathrm{mM} \mathrm{NaF}, 30 \mathrm{mM}$ sodium pyrophosphate, $1 \mathrm{mM} \mathrm{Na} 3 \mathrm{VO} 4,10 \mathrm{mM}$ 2-glycerophosphate, $10 \mu \mathrm{g} / \mathrm{mL}$ leupeptin, $5 \mu \mathrm{g} / \mathrm{mL}$ aprotinin, $5 \mu \mathrm{g} / \mathrm{mL}$ pepstatin, and
$20 \mathrm{mM}$ microcystin-LR. Immunoblotting was done using the following primary antibodies: rabbit polyclonal ZC3H18 (1:1000, A304-682A, Bethyl Laboratories Inc.); mouse monoclonal BRCA1 (1:2000, sc-6954, Santa Cruz Biotechnology); mouse monoclonal E2F1 (1:500, ab4070, Abcam); rabbit polyclonal E2F4 (1:1000, NBP1-21374, Novus Biologicals); mouse monoclonal DNMT1 (1:5000, ab13537, Abcam); mouse monoclonal HSP90 (D. Toft, Mayo Clinic, H9010), and rabbit monoclonal HA-tag (1:1000, CST-3724S, Cell Signaling Technology). Secondary antibodies used were: horseradish peroxidase-conjugated anti-mouse immunoglobulin G (1:2000 for BRCA1 and 1:10,000 for all other primary antibodies, 7076 S, Cell Signaling Technology) and anti-rabbit immunoglobulin G (1:16,000 for ZC3H18 and 1:10,000 for all other primary antibodies, 7074 S, Cell Signaling Technology)

HR assays. HR assays were performed using OVCAR-8-DR-GFP cells ${ }^{42}$. The cells were transfected twice. On day 1 , they were transfected with siRNAs only. On day 2 , they were transfected with the same siRNAs as on day 1 along with $20 \mu \mathrm{g}$ pCßASceI plasmid (encoding I-SceI) with empty vector (pcDNA3) or expression 
vectors for SFB-ZC3H18 or HA-BRCA1. GFP fluorescence was assessed by flow cytometry on day 5 .

\section{RNA extraction, cDNA synthesis, and quantitative real-time PCR (qPCR)}

Total RNA was extracted from frozen cells using the miRNeasy mini kit (Qiagen) following the supplier's instructions. The extracted RNA was converted to cDNA using oligo(dT) primers and SuperScript ${ }^{\text {wI }}$ III reverse transcriptase (ThermoFisher Scientific). Quantitative PCR was performed in triplicate for each sample on a CFX96 real-time PCR system (Bio-Rad) using iTaq Universal SYBR Green Supermix (Bio-Rad). Expression was normalized to GAPDH. The qPCR primers used were:

BRCA1:

Forward: 5'-GCCAAGGCAAGATCTAGAGG-3'

Reverse: 5'-GTTGCCAACACGAGCTGA-3'

ZC3H18:

Forward: 5'-TCCCGGTCTTCATCCTACAG-3' Reverse: $5^{\prime}$-CCGGCTTCTTCACTGACTTC-3' E2F4:

Forward: 5'-CATAGGGGGCAGTGTCTTGT-3' Reverse: $5^{\prime}$-CTAAAGGCCCAGCAGAAGTG-3' $\mathrm{ZCCHC8}$ :

Forward: 5'-CTGGCCGGTGCATATAACTT-3' Reverse: 5'-TGTACCACCACACCTGGCTA-3' GAPDH:

Forward: 5'-GAAGGTGAAGGTCGGAGTCA-3'

Reverse: 5'-AATGAAGGGGTCATTGATGG-3'

Bisulfite sequencing. Cells were harvested $48 \mathrm{~h}$ after siRNA transfection, and genomic DNA was extracted using phenol-chloroform. Genomic DNA $(2 \mu \mathrm{g})$ was modified with bisulfite using the EpiMark ${ }^{\circledR}$ Bisulfite Conversion Kit (New England Biolabs) according to the supplier's protocol. Bisulfite-modified DNA (40 ng) was amplified by PCR using Platinum ${ }^{\text {sut }}$ Taq DNA polymerase and primer pairs (see below) to cover the BRCA1 promoter (GenBank Accession No. U37574). The PCR products were run on a $1 \%$ agarose gel, excised, extracted using QIAquick Gel Extraction Kit (Qiagen), and subcloned into $\mathrm{pCR}^{\mathrm{m}}$ 2.1-TOPO ${ }^{\circledR} \mathrm{TA}$ vector using TOPO $^{\circledast}$ TA Cloning ${ }^{\oplus}$ Kit (ThermoFisher Scientific). At least 10 clones of each PCR product were subjected to Sanger sequencing, and $\mathrm{CpG}$ methylation was analyzed by Quantification Tool for Methylation Analysis (QUMA) ${ }^{48}$. PCR primers specific for bisulfite-converted BRCA1 promoter were designed using MethPrimer soft-

ware ${ }^{49}$. The positions of the primers are shown in Fig. 3a.

Promoter region 1422-1967:

Forward: 5'-AGATTGGGTGGTTAATTTAGAGTTT- ${ }^{\prime \prime}$

Reverse: $5^{\prime}$-ATAATATCCCCCTCAAAACATATTC-3'

The reverse primer was used for Sanger sequencing.

Chromatin immunoprecipitation (ChIP) assays. Cells $\left(1 \times 10^{7}\right)$ in $15-\mathrm{cm}$ dishes were cross-linked with $1 \%$ formaldehyde in media for $10 \mathrm{~min}$ at room temperature, and the unreacted formaldehyde was quenched by the addition of one-tenth volume of $1.25 \mathrm{M}$ glycine ( $\mathrm{pH}$ 7.0). The cells were washed with PBS, dislodged by scraping, collected by centrifugation at $800 \times g$ for $5 \mathrm{~min}$ at $4{ }^{\circ} \mathrm{C}$, resuspended in cell lysis buffer (10 mM Tris HCl, pH 7.5, $10 \mathrm{mM} \mathrm{NaCl}, 0.5 \% \mathrm{NP}-40)$, and incubated on ice for $15 \mathrm{~min}$. The pellet (chromatin) was digested with micrococcal nuclease ( 2.5 units/ml; New England Biolabs) for 20 min at $37^{\circ} \mathrm{C}$ and sonicated for 15 min using the Diagenode Bioruptor-300 sonication system. Aliquots of sheared chromatin were immunoprecipitated using protein G Dynabeads ${ }^{\mathrm{mw}}$ and $1 \mu \mathrm{g}$ of the above-mentioned ZC3H18, DNMT1, E2F1, or E2F4 antibodies. Immunoprecipitation with normal mouse IgG ( $1 \mu \mathrm{g} / \mathrm{ChIP}, 0107-01$, SouthernBiotech) or rabbit IgG (1 $\mu \mathrm{g} / \mathrm{ChIP}, 0111-01$, SouthernBiotech) were used as negative controls. After immunoprecipitation, crosslinks were reversed by heating to $60^{\circ} \mathrm{C}$, and immunoprecipitated DNA was purified using spin columns (Cat. No. 11732676001 , Roche). qPCR analysis of the ChIP and genomic input DNAs was performed using iQ $^{\text {mit }}$ SYBR $^{\circledast}$ Green Supermix (Bio-Rad) using the supplier's protocol. The following primers that amplify the BRCA1 promoter region that contains the E2FA and E2FB sites were used: forward, 5'-CTTGATTTCGTATTCTGAGAGG-3' and reverse, 5'GCTGTGGGGTTTCTCAGATA-3'. For the ChIP-Re-ChIP assay, ChIP assays were first performed as described above, except that each ChIP was done in duplicate and eluates from the duplicate ChIPs were pooled to enhance the signals obtained in the second ChIP. Forty microliters (1/5th volume) from the first ChIP was used for subsequent analysis of the first immunoprecipitation. The remaining solution was diluted in $500 \mu \mathrm{L}$ of IP dilution buffer $(0.01 \%$ SDS, $1.1 \%$ Triton X-100, $16.7 \mathrm{mM}$ Tris- $\mathrm{HCl}$ at $\mathrm{pH} 8.1,167 \mathrm{mM} \mathrm{NaCl}$ ) and immunoprecipitation was performed using the second antibody, and the immunoprecipitated DNA was subsequently analyzed by qPCR as described above.

Protein purification. To produce recombinant full-length ZC3H18 protein, E. coli strain BL21(DE3) transformed with the SFB-ZC3H18-pET24a(+) construct was cultured in $250 \mathrm{ml} \mathrm{LB}$ medium at $37^{\circ} \mathrm{C}$ until it reached an OD600 between 0.5 and 0.8 . The culture was transferred to a $16^{\circ} \mathrm{C}$ shaking incubator, $0.5 \mathrm{mM}$ isopropyl- $\beta$ D-thiogalactopyranoside (IPTG) was added, and the culture was incubated overnight. The cells were harvested by centrifugation, resuspended in PB $(50 \mathrm{mM}$ sodium phosphate, $\mathrm{pH} 7.4,300 \mathrm{mM} \mathrm{NaCl}$ ) containing $1 \mathrm{mM}$ imidazole and sonicated on ice. The lysates were cleared by centrifugation at $14,000 \times g$ for $30 \mathrm{~min}$ at $4{ }^{\circ} \mathrm{C}$ and incubated with Ni-NTA His-Bind Superflow beads (Novagen) for $1 \mathrm{~h}$ at $4{ }^{\circ} \mathrm{C}$. The beads were washed 3 times with $\mathrm{PB}$ containing $1 \mathrm{mM}$ imadazole and eluted with $\mathrm{PB}$ containing $150 \mathrm{mM}$ imidazole $\left(\mathrm{pH} \mathrm{7.4)}\right.$ at $4{ }^{\circ} \mathrm{C}$. The eluate was then exchanged into NETN buffer ( $20 \mathrm{mM}$ Tris- $\mathrm{HCl}, \mathrm{pH} 8.0,100 \mathrm{mM} \mathrm{NaCl}, 0.5 \% \mathrm{NP}-$ 40 , and $1 \mathrm{mM}$ EDTA) using an Amicon ${ }^{\circledR}$ Ultra-15 centrifugal filter unit system (Sigma) and incubated with anti-FLAG M2 antibody (F3165, Sigma) and protein G (ThermoFisher Scientific) for $2 \mathrm{~h}$ at $4{ }^{\circ} \mathrm{C}$. The beads were washed five times with NETN buffer (20 mM Tris-HCl, pH 8.0, $100 \mathrm{mM} \mathrm{NaCl}, 0.5 \% \mathrm{NP}-40$, and $1 \mathrm{mM}$ EDTA), and eluted with $20 \mu \mathrm{g}$ 3xFLAG peptide (Sigma) by centrifugation at $2000 \times g$ for $3 \mathrm{~min}$ at $4{ }^{\circ} \mathrm{C}$.

To produce E2F4 and E2F1 proteins, pSFB-E2F4 or pSFB-E2F1 plasmids $(40 \mu \mathrm{g} /$ transfection) were electroporated into K562 cells. Cells were harvested $24 \mathrm{~h}$ after transfection, and lysed with RIPA buffer $(150 \mathrm{mM} \mathrm{NaCl}, 1 \% \mathrm{NP}-40,0.5 \%$ sodium deoxycholate, $0.1 \% \mathrm{SDS}$, and $50 \mathrm{mM}$ Tris, $\mathrm{pH}$ 8.0). The clarified lysate was incubated with anti-FLAG M2 antibody and protein $\mathrm{G}$ agarose affinity resin (ThermoFisher Scientific) for $4 \mathrm{~h}$, washed 5 times in RIPA buffer, and SFB-tagged proteins were eluted with $20 \mu \mathrm{g}$ 3xFLAG peptide in elution buffer $(50 \mathrm{mM}$ Tris, pH7.4, $5 \mathrm{mM} \mathrm{MgCl} 2,150 \mathrm{mM} \mathrm{NaCl})$. The purity of the eluted proteins was determined by Coomassie Blue (Bio-Rad) or SYPRO ${ }^{\circledR}$ Ruby staining (ThermoFisher Scientific) in accordance with supplier's instructions.

Electrophoretic mobility gel-shift assay. Complementary 50-bp single-stranded oligonucleotides corresponding to the BRCA1 promoter with wild-type E2FA and E2FB sites or mutations in E2FA, E2FB, or both E2FA and E2FB sites were synthesized by Integrated DNA Technologies. The complementary oligonucleotides were end-labeled with $\gamma_{-}{ }^{32} \mathrm{P}-\mathrm{ATP}$ using polynucleotide kinase (NEB), and purified using CHROMA SPIN ${ }^{\mathrm{TM}}+\mathrm{TE}-10$ columns (Takara). Equimolar concentrations of each oligonucleotide were diluted into annealing buffer $(20 \mathrm{mM}$ Tris- $\mathrm{HCl}, \mathrm{pH} 8.0$, $50 \mathrm{mM} \mathrm{NaCl}, 1 \mathrm{mM} \mathrm{MgCl} 2$ ) in a microcentrifuge tube, heated to $100^{\circ} \mathrm{C}$ in a water bath, and cooled slowly to $4{ }^{\circ} \mathrm{C}$ to anneal the oligonucleotides. EMSA assays were performed by incubating the duplexed oligonucleotides in gel-shift buffer $(20 \mathrm{mM}$ Tris- $\mathrm{HCl}, \mathrm{pH} 8.0,50 \mathrm{mM} \mathrm{NaCl}, 1 \mathrm{mM} \mathrm{MgCl}, 5 \mathrm{mg} / \mathrm{ml} \mathrm{BSA}$, and $1 \mathrm{mM}$ dithiothreitol) and various concentrations of purified ZC3H18, E2F1, and/or E2F4 proteins for $20 \mathrm{~min}$ at room temperature. For cold competition, the binding reaction mixture was pre-incubated with 50-, 200-, or 500-fold excess unlabeled probe for $10 \mathrm{~min}$ before adding the labeled probe. For supershift assays, the reaction mixture was pre-incubated with anti-S-tag antibody ${ }^{50}$, which recognizes the N-terminal S-peptide of the SFB-tagged proteins, for $30 \mathrm{~min}$ on ice before adding the labeled DNA probe. Samples were run on a $5 \%$ native acrylamide gel in $0.25 \times \mathrm{TBE}(0.0225 \mathrm{M}$ Tris-borate, $0.0005 \mathrm{M}$ EDTA pH 8.0$)$ at $4{ }^{\circ} \mathrm{C}$. The gel was dried, and autoradiography was performed.

BRCA1 splicing analysis. Fourty-eight hour after siRNA transfection, total RNA was extracted and cDNA was synthesized as described above. The cDNA was amplified by PCR using Platinum ${ }^{\text {ma }}$ Taq DNA polymerase and primer pairs ${ }^{25}$ to cover all the BRCA1 exons.

The primers used were: Exon 1A-3: forward, 5'-GACAGGCTGTGGGGTTT CT- $3^{\prime}$

reverse, 5'-TTTGTGGAGACAGGTTCCTTGA-3'

Exon 1A-6: forward, 5'-GACAGGCTGTGGGGTTTCT-3';

reverse, ${ }^{\prime}$-TCCAAACCTGTGTCAAGCTG-3

Exon 1A-11q: forward, 5'-GACAGGCTGTGGGGTTTCT-3'; reverse, 5'-TGG CTCCACATGCAAGTTTG-3'

Exon 7-11q: forward, 5'-CATCCAAAGTATGGGCTACAG-3'; reverse, 5'-TGG CTCCACATGCAAGTTTG-3'

Exon 7-12: forward, 5'-CATCCAAAGTATGGGCTACAG-3'; reverse, 5'-CTGAGAGGATAGCCCTGA-3'

Exon 8-13: forward, 5'-GGTTGTATCCGCTGCTTTGT-3'; reverse, 5' - ATGGAAGGGTAGCTGTTAGAAGG-3'

Exon 10-12: forward, 5'-CCAGGGATGAAATCAGTTTGG-3'; reverse, 5'-GCGTCTCTGAAGACTGCTCA-3'

Exon 10-13: forward, 5'-CCAGGGATGAAATCAGTTTGG-3'; reverse, 5'-ATGGAAGGGTAGCTGTTAGAAGG-3'

Exon 12-13: forward, 5'-GCGTCTCTGAAGACTGCTCA-3'; reverse, 5'-ATGGGAGCCAGCCTTCTAAC-3

Exon 12-14: forward, 5'-GCGTCTCTGAAGACTGCTCA-3'; reverse, 5'-AAAGGCCTTCTGGATTCTGG-3'

Exon 12-16: forward, 5'-GCGTCTCTGAAGACTGCTCA-3'; reverse, 5'-CTCACACTTTCTTCCATTGC-3'

Exon 13-22: forward, 5'-ATGGGAGCCAGCCTTCTAAC-3'; reverse, 5'-CACAGCTGTACCATCCATTC- ${ }^{\prime}$ Exon 14-22: forward, 5'-TCTGCAGATAGTTCTACCAG-3'; reverse, 5'-CACAGCTGTACCATCCATTC-3'

Exon 16-22: forward, 5'-AAAGAATGTCCATGGTGGTG-3'; reverse, 5'-CACAGCTGTACCATCCATTC-3'

Exon 16-24: forward, 5'-AAAGAATGTCCATGGTGGTG-3'; reverse, 5'- ACCACAGGTGCCTCACACAT-3' 
Exon 20-24: forward, 5' - AGAAACCACCAAGGTCCAAAG-3';

reverse, 5' - ACCACAGGTGCCTCACACAT-3

The PCR products were run on a $1 \%$ agarose gel to visualize the alternative $B R C A 1$ splicing pattern.

Luciferase reporter assay. OVCAR- 8 cells were transfected with BRCA1-promoter firefly luciferase constructs $(2 \mu \mathrm{g} /$ transfection $)$ and an internal control for transfection efficiency (pRL-SV40 Renilla luciferase reporter construct, Promega $100 \mathrm{ng} /$ transfection) and plated into 6-well plates. Samples were harvested $24 \mathrm{~h}$ after transfection, and luciferase activity was measured using Dual-Glo ${ }^{\circledast}$ luciferase assay system (Promega) following the supplier's protocol. To control for intersample variations in transfection efficiencies, firefly luciferase readouts were normalized to renilla luciferase readouts.

Ex vivo culture of HGSOC tumor tissues from PDX mouse models. To obtain short-term, 2D, ex vivo monolayer cultures of tumor cells, HGSOC tissues from PDX mouse models were harvested, minced into 2-4-mm pieces with a sterile scalpel blade, and dissociated using a tumor dissociation kit (Cat. \# 130-096-730, Miltenyi Biotec) following the supplier's protocol. After dissociation, the cells were washed five times with RPMI-1640 medium (Invitrogen) supplemented with $10 \%$ fetal bovine serum (Invitrogen), 100 units/mL penicillin and 100 units/mL streptomycin (Invitrogen), resuspended in RPMI-1640 medium with 10\% fetal bovine serum without antibiotics, and electroporated with control luciferase (Luc), E2F1, or E2F4 siRNAs as described ${ }^{42}$. The cells were then plated in 24 -well plates in RPMI-1640 supplemented with $10 \%$ fetal bovine serum and 100 units/mL penicillin and 100 units/mL streptomycin (Invitrogen). The plates were cultured for 48 $h$ and harvested for RNA extraction and qRT-PCR.

Analyses of patient and PDX tumors. Fresh tissues from high-grade serous ovarian, primary peritoneal, or fallopian tube cancers were collected at the time of primary debulking surgery at Mayo Clinic, Rochester from chemotherapy naïve patients who provided written and informed consent. All biospecimens were coded with a patient heterotransplant $(\mathrm{PH})$ number to protect patient identity in accordance with the Mayo Clinic Institutional Review Board and in accordance with the Health Insurance Portability and Accountability Act regulations through the Mayo Clinic Ovarian Tumor Repository. PDX models were developed as previously described by intraperitoneal injection into female SCID beige mice (C.B17/IcrHsd-Prkdc scid $L y s t^{b g-J}$; ENVIGO), also in accordance with the Mayo Clinic Institutional Animal Care and Use Committee. Briefly, 0.1 to $0.3 \mathrm{cc}$ of minced fresh patient tumor was mixed 1:1 with McCoy's media with rituximab ${ }^{51}$ in a $1-\mathrm{mL}$ syringe and injected intraperitoneally through a 0.5 -inch 16 -gauge needle. No enzymatic or mechanical tumor dissociation was performed. Mice were monitored by routine palpation for engraftment and when moribund, tumors were snap frozen for subsequent studies. For primary patient samples, surplus tumor tissue in excess of requirements to generate PDXs was also snap frozen for future RNA work.

Total RNA was isolated from tissues collected from 97 patients and 138 nonoverlapping PDX from mice using the RNeasy Micro kit (Qiagen, \#74004) according to the manufacturer instructions. Purification of total RNA concentration and purity was determined on a Thermo Scientific NanoDrop 2000c UV-Vis Spectrophotometer (Thermo Scientific, Wilmington, DE). All samples met RNA integrity number and validated Agilent (Agilent Technologies, Santa Clara, CA) criteria

RNA libraries were prepared according to the manufacturer's instructions for the TruSeq RNA Sample Prep Kit (Illumina, San Diego, CA, USA). The concentration and size distribution of the libraries were determined on an Agilent Bioanalyzer DNA 1000 chip (Santa Clara, CA, USA). Libraries were loaded onto flow cells at concentrations of $8-10$ pM to generate cluster densities of 700,000/ $\mathrm{mm}^{2}$ following Illumina's standard protocol using the Illumina $\mathrm{cBot}$ and $\mathrm{cBot}$ Paired End cluster kit version 3. The flow cells were sequenced as $51 \times 2$ Paired End reads on an Illumina HiSeq 2000 using TruSeq SBS sequencing kit version 3 and SCS version 1.4.8 data collection software. Base calling was performed using Illumina's RTA version 1.12.4.2. There were $\sim 45$ million reads per sample mapped to the human genome, and 21,686 genes were detected. mRNA levels are expressed as RPKM (reads per kilobase per million mapped reads) using the formula: $\left(10^{9} \times\right.$ count $) /$ (total reads $\times$ feature length), where count is the number of reads mapping to the gene or exon, total reads is the total number of reads mapping to all genes or exons in that sample, and feature length is the length of the gene or exon. Spearman correlation was used to assess correlation between BRCA1 mRNA levels with $\mathrm{ZC} 3 \mathrm{H} 18$ and E2F4 mRNA.

RNA-seq analyses of OVCAR-8 cells. Forty-eight hour after control luciferase, ZC3H18 \#1, or ZC3H18 \#2 siRNA transfection, RNA was isolated using miRNeasy mini kit (Qiagen). Three independent RNA samples were prepared for each of the transfected siRNAs. Libraries were prepared (Illumina TruSeq mRNA v2) and processed through Mayo Clinic's MAP-RSeq (v2.1.0) application ${ }^{52}$. The gene counts were generated by FeatureCounts ${ }^{53}$ using Ensembl's hg19 gene definition file. RSeqQC ${ }^{54}$ was used to create quality control metrics, including gene body coverage plots, to insure the results from each sample were reliable and could be collectively used for a differential expression analysis. Genes with an average of $<25$ reads were removed from the differential expression analysis. The $\mathrm{R}$ package (v3.3.1), edge $\mathrm{R}^{55}$ was used to identify which genes were differentially expressed. Statistically significant genes were defined by having a false discovery rate below 0.5 . These differentially expressed genes were then used in a hypergeometric gene set enrichment test to evaluate enrichment in publically defined homologous recombination genes. The publically defined homologous recombination genes were defined by $\mathrm{KEGG}^{24}$ and the Gene Ontology ${ }^{56}$.

In vivo PARPi efficacy studies. OVCAR-8 cells with doxycycline-inducible control non-targeting or $\mathrm{ZC} 3 \mathrm{H} 18$ shRNAs were produced by lentiviral transduction. OVCAR- 8 cells were seeded at $5 \times 10^{4}$ cells/well in six-well plates with $2 \mathrm{~mL}$ of complete medium (RPMI-1640 supplemented with $8 \%$ FBS) and cultured overnight at $37^{\circ} \mathrm{C}$. The culture medium was replaced with $1: 1$ mixture of complete medium containing $2 \mu \mathrm{g} / \mathrm{mL}$ polybrene and TRIPZ doxycycline-inducible nontargeting shRNA control (shNT; Cat. No. RHS4743, Dharmacon) or

ZC3H18 shRNA (shZC3, Cat. No. V2THS_19374, Dharmacon) lentiviral particle stocks. After $48 \mathrm{~h}$, the medium was replaced with complete medium containing $0.75 \mu \mathrm{g} / \mathrm{mL}$ puromycin to select for stable shNT and shZC3 genomically integrated populations.

All animal studies and procedures were reviewed and approved by the Mayo Clinic Institutional Animal Care and Use Committee (IACUC).

Immunocompromised 8-week-old SCID Beige female mice (C.B-17/IcrHsdPrkdc scid $L y s t^{b g-J}$; ENVIGO) were inoculated with $5 \times 10^{6}$ stable shNT or shZC3 OVCAR-8 cells intraperitonially in $200 \mu \mathrm{L}$ serum-free RPMI-1640 medium. After 5 days, mice were given doxycycline chow (ENVIGO), which was continued until the end of the xenograft study. After 3 days, mice inoculated with shNT- or shZC3-transduced cells were assigned randomly into two groups (10 mice per group) that received vehicle control ( $0.5 \%$ methyl cellulose) or $50 \mathrm{mg} / \mathrm{kg}$ olaparib daily by oral gavage. After 28 days of treatment, tumors were weighed, snap frozen in liquid $\mathrm{N}_{2}$, and ground with mortar and pestal. In total, $10 \mathrm{mg}$ of tumor tissue was then lysed in SDS-PAGE sample buffer, and protein concentrations were determined by Coomassie Blue staining of pilot SDS-PAGE gels. Equal protein concentrations from each tumor were then immunoblotted for ZC3H18, BRCA1, and HSP90.

16q24.2 copy number and ZC3H18 and BRCA1 alterations in HGSOC. Analysis of 16q24.2 copy number changes in HGSOC was performed using the GDC TCGA Ovarian Cancer dataset and the UCSC Xena Browser (https://xenabrowser.net) ${ }^{57}$.

Potential mutual exclusivity analysis of $B R C A 1$ deletions and driver mutations versus $\mathrm{ZC} 3 \mathrm{H} 18$ deletions and low $\mathrm{ZC} 3 \mathrm{H} 18 \mathrm{mRNA}$ levels was performed using genomics data in the cBioPortal (https://www.cbioportal.org) ${ }^{58,59}$. ZC $3 \mathrm{H} 18$ deep (homologous) deletions and mRNA expression $<2$ standard deviations below mean versus deep (homologous) deletions and driver mutations for BRCA1 in ovarian serous cystadenocarcinoma and breast invasive carcinoma were compared using TCGA PanCancer Atlas databases. OncoPrints of the alterations generated in the cBioPortal are presented.

Statistics. Data in bar and line graphs are reported as means \pm SEM. Statistical analyses were performed in GraphPad Prism Version 8.0 (GraphPad Software, La Jolla, CA, USA) with unpaired $t$-test. Spearman correlations between $Z$ C $3 H 18$ versus BRCA1 and E2F4 versus BRCA1 mRNA expression in HGSOC PDX and patient tumors (Fig. 7a) were performed in R version 3.4 .2 (https://www.R-project. org/). The following depicts statistical significance: ${ }^{*} p<0.05,{ }^{* *} p<0.01,{ }^{* *} p<$ 0.001 .

Reporting summary. Further information on research design is available in the Nature Research Reporting Summary linked to this article.

\section{Data availability}

RNA-seq data have been deposited in the GEO accession GSE136533 and also provided as Supplementary Data 1 and 2. The authors declare that all other data supporting the findings of this study are available within the paper and its supplementary information and source data files upon reasonable request.

Received: 2 August 2018; Accepted: 18 September 2019; Published online: 11 October 2019

\section{References}

1. Konstantinopoulos, P. A., Ceccaldi, R., Shapiro, G. I. \& D’Andrea, A. D. Homologous recombination deficiency: exploiting the fundamental vulnerability of ovarian cancer. Cancer Discov. 5, 1137-1154 (2015)

2. Ledermann, J. A., Drew, Y. \& Kristeleit, R. S. Homologous recombination deficiency and ovarian cancer. Eur. J. Cancer 60, 49-58 (2016). 
3. Norquist, B. M. et al. Mutations in homologous recombination genes and outcomes in ovarian carcinoma patients in GOG 218: an NRG oncology/ gynecologic oncology group study. Clin. Cancer Res. 24, 777-783 (2018).

4. Nesic, K., Wakefield, M., Kondrashova, O., Scott, C. L. \& McNeish, I. A. Targeting DNA repair: the genome as a potential biomarker. J. Pathol. 244, 586-597 (2018).

5. Swisher, E. M. et al. Methylation and protein expression of DNA repair genes: association with chemotherapy exposure and survival in sporadic ovarian and peritoneal carcinomas. Mol. Cancer 8, 48 (2009).

6. Esteller, M. et al. Promoter hypermethylation and BRCA1 inactivation in sporadic breast and ovarian tumors. J. Natl. Cancer Inst. 92, 564-569 (2000).

7. Ruscito, I. et al. BRCA1 gene promoter methylation status in high-grade serous ovarian cancer patients-a study of the tumour Bank ovarian cancer (TOC) and ovarian cancer diagnosis consortium (OVCAD). Eur. J. Cancer 50, 2090-2098 (2014).

8. Bai, X. F. et al. BRCA1 promoter hypermethylation in sporadic epithelial ovarian carcinoma: Association with low expression of BRCA1, improved survival and co-expression of DNA methyltransferases. Oncol. Lett. 7, 1088-1096 (2014).

9. Swisher, E. M. et al. Rucaparib in relapsed, platinum-sensitive high-grade ovarian carcinoma (ARIEL2 Part 1): an international, multicentre, open-label, phase 2 trial. Lancet Oncol. 18, 75-87 (2017).

10. Prieske, K. et al. Loss of BRCA1 promotor hypermethylation in recurrent high-grade ovarian cancer. Oncotarget 8, 83063-83074 (2017).

11. Cancer Genome Atlas Research Network. Integrated genomic analyses of ovarian carcinoma. Nature 474, 609-615 (2011).

12. Joshi, P. M., Sutor, S. L., Huntoon, C. J. \& Karnitz, L. M. Ovarian cancerassociated mutations disable catalytic activity of CDK12, a kinase that promotes homologous recombination repair and resistance to cisplatin and poly(ADP-ribose) polymerase inhibitors. J. Biol. Chem. 289, 9247-9253 (2014).

13. Blazek, D. et al. The Cyclin K/Cdk12 complex maintains genomic stability via regulation of expression of DNA damage response genes. Gene Dev. 25, 2158-2172 (2011).

14. Mueller, C. R. \& Roskelley, C. D. Regulation of BRCA1 expression and its relationship to sporadic breast cancer. Breast Cancer Res. 5, 45-52 (2003).

15. Bindra, R. S. et al. Hypoxia-induced down-regulation of BRCA1 expression by E2Fs. Cancer Res. 65, 11597-11604 (2005).

16. De Siervi, A. et al. Transcriptional autoregulation by BRCA1. Cancer Res. 70, 532-542 (2010).

17. Winczura, K. et al. Characterizing $\mathrm{ZC} 3 \mathrm{H} 18$, a multi-domain protein at the interface of RNA production and destruction decisions. Cell Rep. 22, 44-58 (2018).

18. Chi, B. et al. A sub-element in PRE enhances nuclear export of intronless mRNAs by recruiting the TREX complex via ZC3H18. Nucleic Acids Res. 42, 7305-7318 (2014).

19. Andersen, P. R. et al. The human cap-binding complex is functionally connected to the nuclear RNA exosome. Nat. Struct. Mol. Biol. 20, 1367-1376 (2013).

20. Giacometti, S. et al. Mutually exclusive CBC-containing complexes contribute to RNA fate. Cell Rep. 18, 2635-2650 (2017).

21. Engler, D. A. et al. Genome wide DNA copy number analysis of serous type ovarian carcinomas identifies genetic markers predictive of clinical outcome. PLoS ONE 7, e30996 (2012).

22. Wrzeszczynski, K. O. et al. Identification of tumor suppressors and oncogenes from genomic and epigenetic features in ovarian cancer. PLoS ONE 6, e28503 (2011).

23. Nakanishi, K., Cavallo, F., Brunet, E. \& Jasin, M. Homologous recombination assay for interstrand cross-link repair. Methods Mol. Biol. 745, 283-291 (2011).

24. Kanehisa, M., Sato, Y., Kawashima, M., Furumichi, M. \& Tanabe, M. KEGG as a reference resource for gene and protein annotation. Nucleic Acids Res. 44, D457-D462 (2016).

25. Colombo, M. et al. Comprehensive annotation of splice junctions supports pervasive alternative splicing at the BRCA1 locus: a report from the ENIGMA consortium. Hum. Mol. Genet. 23, 3666-3680 (2014).

26. Ignatov, T. et al. BRCA1 promoter methylation is a marker of better response to platinum-taxane-based therapy in sporadic epithelial ovarian cancer. J. Cancer Res. Clin. Oncol. 140, 1457-1463 (2014).

27. Hansmann, T. et al. Constitutive promoter methylation of BRCA1 and RAD51C in patients with familial ovarian cancer and early-onset sporadic breast cancer. Hum. Mol. Genet. 21, 4669-4679 (2012).

28. Stordal, B. et al. BRCA1/2 mutation analysis in 41 ovarian cell lines reveals only one functionally deleterious BRCA1 mutation. Mol. Oncol. 7, 567-579 (2013).

29. Lee, B. K., Bhinge, A. A. \& Iyer, V. R. Wide-ranging functions of E2F4 in transcriptional activation and repression revealed by genome-wide analysis. Nucleic Acids Res. 39, 3558-3573 (2011).
30. Oberley, M. J., Inman, D. R. \& Farnham, P. J. E2F6 negatively regulates BRCA1 in human cancer cells without methylation of histone $\mathrm{H} 3$ on lysine 9. J. Biol. Chem. 278, 42466-42476 (2003).

31. Swiss, V. A. \& Casaccia, P. Cell-context specific role of the E2F/Rb pathway in development and disease. Glia 58, 377-390 (2010).

32. Bindra, R. S. \& Glazer, P. M. Basal repression of BRCA1 by multiple E2Fs and pocket proteins at adjacent E2F sites. Cancer Biol. Ther. 5, 1400-1407 (2006).

33. Wang, A., Schneider-Broussard, R., Kumar, A. P., MacLeod, M. C. \& Johnson, D. G. Regulation of BRCA1 expression by the Rb-E2F pathway. J. Biol. Chem. 275, 4532-4536 (2000).

34. Robertson, K. D. et al. DNMT1 forms a complex with Rb, E2F1 and HDAC1 and represses transcription from E2F-responsive promoters. Nat. Genet. 25, 338-342 (2000).

35. Truax, A. D. \& Greer, S. F. ChIP and Re-ChIP assays: investigating interactions between regulatory proteins, histone modifications, and the DNA sequences to which they bind. Methods Mol. Biol. 809, 175-188 (2012).

36. Shilpa, V. et al. BRCA1 promoter hypermethylation and protein expression in ovarian carcinoma-an Indian study. Tumour Biol. 35, 4277-4284 (2014).

37. Wang, Y. Q. et al. Epigenetic inactivation of BRCA1 through promoter hypermethylation in ovarian cancer progression. J. Obstet. Gynaecol. Res. 39, 549-554 (2013).

38. Attwooll, C., Denchi, E. L. \& Helin, K. The E2F family: specific functions and overlapping interests. EMBO J. 23, 4709-4716 (2004).

39. Hsu, J. \& Sage, J. Novel functions for the transcription factor E2F4 in development and disease. Cell Cycle 15, 3183-3190 (2016)

40. Ren, B. et al. E2F integrates cell cycle progression with DNA repair, replication, and G(2)/M checkpoints. Genes Dev. 16, 245-256 (2002).

41. Gewurz, B. E. et al. Genome-wide siRNA screen for mediators of NF-kappaB activation. Proc. Natl Acad. Sci. USA 109, 2467-2472 (2012).

42. Huntoon, C. J. et al. ATR inhibition broadly sensitizes ovarian cancer cells to chemotherapy independent of BRCA status. Cancer Res. 73, 3683-3691 (2013).

43. Sardet, C. et al. E2F-4 and E2F-5, two members of the E2F family, are expressed in the early phases of the cell cycle. Proc. Natl Acad. Sci. USA 92, 2403-2407 (1995)

44. Lukas, J., Petersen, B. O., Holm, K., Bartek, J. \& Helin, K. Deregulated expression of E2F family members induces S-phase entry and overcomes p16INK4A-mediated growth suppression. Mol. Cell. Biol. 16, 1047-1057 (1996).

45. Chini, C. C. \& Chen, J. Repeated phosphopeptide motifs in human Claspin are phosphorylated by Chk1 and mediate Claspin function. J. Biol. Chem. 281, 33276-33282 (2006)

46. Wang, L. et al. BRCA1 is a negative modulator of the PRC2 complex. EMBO J.32, 1584-1597 (2013).

47. Richardson, C., Moynahan, M. E. \& Jasin, M. Double-strand break repair by interchromosomal recombination: suppression of chromosomal translocations. Genes Dev. 12, 3831-3842 (1998).

48. Kumaki, Y., Oda, M. \& Okano, M. QUMA: quantification tool for methylation analysis. Nucleic Acids Res. 36, W170-W175 (2008).

49. Li, L. C. \& Dahiya, R. MethPrimer: designing primers for methylation PCRs. Bioinformatics 18, 1427-1431 (2002).

50. Hackbarth, J. S. et al. S-peptide epitope tagging for protein purification, expression monitoring, and localization in mammalian cells. Biotechniques 37 , 835-839 (2004)

51. Butler, K. A. et al. Prevention of human lymphoproliferative tumor formation in ovarian cancer patient-derived xenografts. Neoplasia 19, 628-636 (2017).

52. Kalari, K.R. et al. MAP-RSeq: Mayo analysis pipeline for RNA sequencing BMC Bioinformatics 15, 224 (2014).

53. Liao, Y., Smyth, G.K. \& Shi, W. featureCounts: an efficient general purpose program for assigning sequence reads to genomic features. Bioinformatics30, 923-930 (2014)

54. Wang, L., Wang, S. \& Li, W. RSeQC: quality control of RNA-seq experiments. Bioinformatics28, 2184-2185 (2012)

55. Robinson, M.D., McCarthy, D.J. \& Smyth, G.K. edgeR: a Bioconductor package for differential expression analysis of digital gene expression data. Bioinformatics26, 139-140 (2010).

56. Ashburner, M. et al. Gene Ontology: tool for the unification of biology. Nat Genet.25, 25-29 (2000)

57. Goldman, M. et al. The UCSC Xena platform for public and private cancer genomics data visualization and interpretation. Preprint at bioRxiv https://doi. org/10.1101/326470 https://www.biorxiv.org/content/10.1101/326470v5 (2019).

58. Gao, J. J. et al. Integrative analysis of complex cancer genomics and clinical profiles using the cBioPortal. Sci Signal 6, pl1 https://www.ncbi.nlm.nih.gov/ pubmed/23550210 (2013) 
59. Cerami, E. et al. The cBio cancer genomics portal: an open platform for exploring multidimensional cancer genomics data. Cancer Discov. 2, 401-404 (2012).

\section{Acknowledgements}

This work was supported by NIH (R01 CA194498 to L.M.K, R01 CA184502 to S.J.W., and P50 CA136393 to S.H.K), a Foundation for Women's Cancer Genentech Ovarian Cancer Young Investigator Career Development Award to A.K., the Fred C. and Katherine Andersen Foundation, a Stand Up To Cancer-Ovarian Cancer Research Fund Alliance-National Ovarian Cancer Coalition Dream Team Translational Research Grant (SU2C-AACR-DT16-15) to L.M.K. and S.H.K., and a Wallace and Evelyn Simmers Career Development Award for Ovarian Cancer Research to A.K. We thank Dr. Keith Robertson and Joyce Thompson for providing the protocol and guidance for bisulfite sequencing. We thank Drs. Somaira Nowsheen and Min Deng for help with recombinant $\mathrm{ZC} 3 \mathrm{H} 18$ protein purification. We thank Dr. Yuichi Machida for allowing us to use his qRT-PCR machine. We also thank Drs. Martin Fernandez-Zapico and Luciana Almada for help with ChIP and luciferase reporter assays. The results shown in Supplementary Fig 10 are in whole based upon data generated by the TCGA Research Network

\section{Author contributions}

A.K., A.L.O., S.J.W., S.H.K. and L.M.K. designed the studies. A.K., C.J.H., X.H. and M.Z performed experiments. A.K., E.P.H., D.R.O. and A.L.O. analyzed data. A.K. and L.M.K. wrote the manuscript with input from all the other authors.

\section{Competing interests}

The authors declare no competing interests.

\section{Additional information}

Supplementary information is available for this paper at https://doi.org/10.1038/s41467019-12610-x.

Correspondence and requests for materials should be addressed to L.M.K.

Peer review information Nature Communications thanks Simon Powell and the other, anonymous, reviewer(s) for their contribution to the peer review of this work.

Reprints and permission information is available at http://www.nature.com/reprints

Publisher's note Springer Nature remains neutral with regard to jurisdictional claims in published maps and institutional affiliations.

(c) (i) Open Access This article is licensed under a Creative Common Attribution 4.0 International License, which permits use, sharing, adaptation, distribution and reproduction in any medium or format, as long as you give appropriate credit to the original author(s) and the source, provide a link to the Creative Commons license, and indicate if changes were made. The images or other third party material in this article are included in the article's Creative Commons license, unless indicated otherwise in a credit line to the material. If material is not included in the article's Creative Commons license and your intended use is not permitted by statutory regulation or exceeds the permitted use, you will need to obtain permission directly from the copyright holder. To view a copy of this license, visit http://creativecommons.org/ licenses/by/4.0/.

(C) The Author(s) 2019 